\title{
Satellite Laser Ranging to Low Earth Orbiters - Orbit and Network Validation
}

\author{
Daniel Arnold · Oliver Montenbruck · Stefan Hackel • Krzysztof Sośnica
}

Received: date / Revised version: date

\begin{abstract}
Satellite laser ranging (SLR) to low Earth orbiters (LEOs) provides optical distance measurements with mm-to cm-level precision. SLR residuals, i.e., differences between measured and modeled ranges, serve as a common figure of merit for the quality assessment of orbits derived by radiometric tracking techniques. We discuss relevant processing standards for the modeling of SLR observations and highlight the importance of line-of-sight dependent range corrections for the various types of laser retroreflector ararys (LRAs). A 1-3 cm consistency of SLR observations and GPSbased precise orbits is demonstrated for a wide range of past and present LEO missions supported by the International Laser Ranging Service (ILRS). A parameter estimation approach is presented to investigate systematic orbit errors and it is shown that SLR validation of LEO satellites is not only able to detect radial but also along-track and cross-track offsets. SLR residual statistics clearly depend on the employed precise orbit determination technique (kinematic vs. reduced-dynamic, float vs. fixed ambiguities) but also reveal pronounced differences in the ILRS station performance. Using the residuals-based parameter estimation approach, corrections to ILRS station coordinates, range bi-
\end{abstract}

Daniel Arnold

Astronomical Institute, University of Bern,

Sidlerstrasse 5

CH-3012 Bern, Switzerland

E-mail: daniel.arnold@aiub.unibe.ch

Oliver Montenbruck, Stefan Hackel

German Space Operations Center

Deutsches Zentrum für Luft- und Raumfahrt

D-82230 Weßling, Germany

E-mail: oliver.montenbruck@dlr.de

Krzysztof Sośnica

Institute of Geodesy and Geoinformatics, Wrocław University of Environmental and Life Sciences

Grunwaldzka 53, 50-357 Wrocław, Poland

E-mail: krzysztof.sosnica@igig.up.wroc.pl ases, and timing offsets are derived. As a result, root-meansquare (RMS) residuals of $5-10 \mathrm{~mm}$ have been achieved over a one-year data arc in 2016 using observations from a subset of high-performance stations and ambiguity-fixed orbits of four LEO missions. As a final contribution, we demonstrate that SLR can not only validate single-satellite orbit solutions but also precise baseline solutions of formation flying missions such as GRACE, TanDEM-X, and Swarm.

Keywords precise orbit determination; satellite laser ranging; laser retroreflector array; GPS; orbit and network validation

\section{Introduction}

The use of global navigation satellite system (GNSS) receivers on board of low Earth orbiters (LEOs) has become standard for precise orbit determination (POD) of these satellites. Post-processing of carrier phase data from dual-frequency GNSS receivers enables a positioning of the satellite with errors in the few $\mathrm{cm}$ range. To date, most of the on-board GNSS receivers track signals from the Global Positioning System (GPS) only.

For POD purposes, GPS observations are most commonly processed in an undifferenced precise point positioning (PPP) mode, even though the double-difference processing of either space baselines or space-ground baselines may be preferred in specific applications (Jäggi et al 2007b). The GPS orbits and, in case of undifferenced processing, the GPS satellite clock corrections are usually introduced as fixed external products. Besides the actual orbit parameters, receiver clock offsets and phase ambiguity parameters need to be estimated. Most commonly, ambiguities are adjusted as floatvalued parameters, even though an improved performance can be achieved when fixing them to integer values. Given 


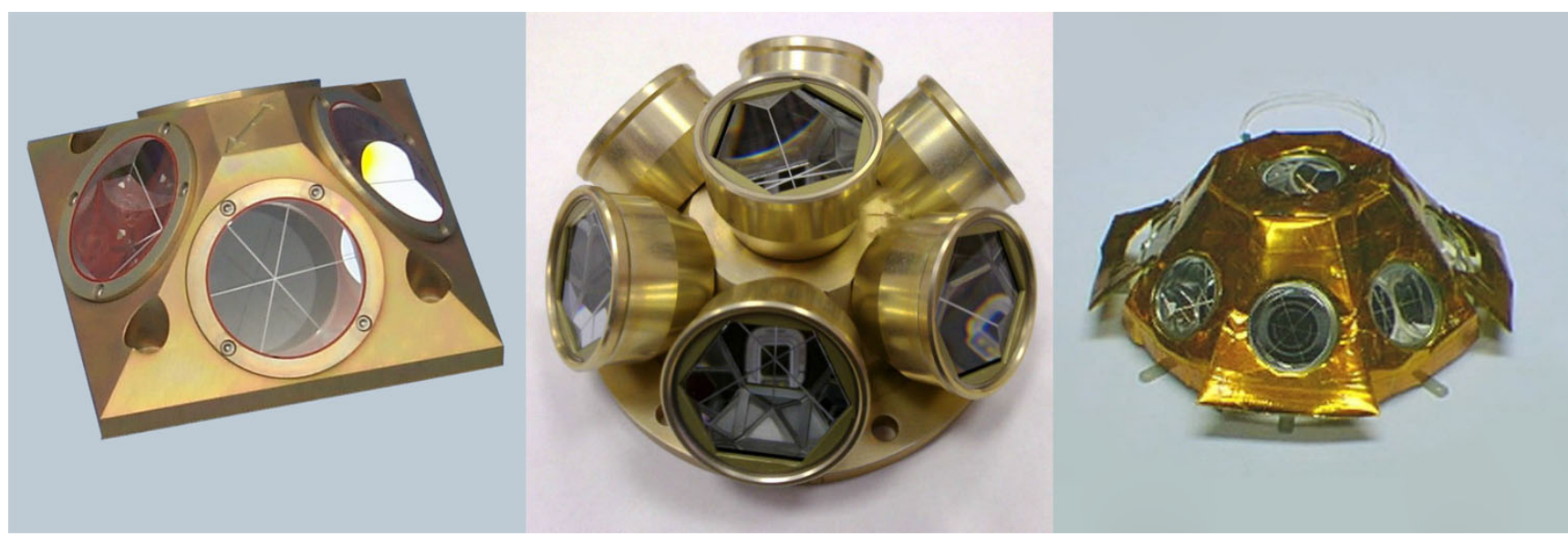

Fig. 1 Laser retroreflector array types used in various LEO missions. Left: CHAMP/GRACE/TerraSAR-X (courtesy GeoForschungsZentrum Potsdam); center: Sentinel-3 (courtesy IPIE/ESA/EUMETSAT); right: Jason-1/2 (courtesy ITE)

the complexity of a traditional double-difference processing, the use of single-receiver ambiguity resolution concepts (Laurichesse et al 2009; Bertiger et al 2010) is gaining more widespread attention for precise orbit determination of LEO satellites.

Generally, three types of LEO POD approaches can be distinguished. In a kinematic POD (Švehla and Rothacher 2002) the GPS data is used for the determination of the 3dimensional satellite position at given observation epochs in a purely geometrical way. This type of orbit is, therefore, fully independent of any force model for the LEO satellite, even though it may indirectly be affected by dynamical models used in the generation of the GPS orbit and clock products. In a purely dynamic POD, on the other hand, the satellite orbit is restricted to obey an equation of motion (Schutz et al 1994). Here, the GPS observations (or other types of tracking data) are used to estimate the initial or boundary conditions for a given orbit arc along with additional force model parameters. In contrast to the kinematic POD this enables the determination of the satellite position (and velocity) also for times at which no tracking data is observed. The performance of a dynamic orbit determination strongly depends on the quality of the gravitational and nongravitational force modeling. Uncertainties in these models render it impossible to exploit the inherent accuracy of the GNSS carrier phase measurements.

The reduced-dynamic POD technique (Wu et al 1991; Yunck et al 1994) combines the advantages of kinematic POD (independence of LEO force models) and dynamic POD (continuous orbit representation governed by physical laws) by introducing different kinds of stochastic parameters in the trajectory model. These parameters may comprise empirical accelerations or instantaneous velocity changes (Tapley et al 2004; Jäggi et al 2006) that are adjusted within the orbit determination and help compensate remaining force model deficiencies. Depending on their a priori constraints and spacing a continuum of orbit solutions in between the purely kinematic and the purely dynamic orbit can be obtained (Jäggi et al 2007a). A proper selection of the stochastic orbit parameters and their constraints allows for the generation of LEO orbit solutions of the highest quality and is nowadays widely applied.

Within this study we demonstrate the use of satellite laser ranging (SLR) for validating GPS-based precise orbit products for LEO satellites and assess the POD performance achieved in various missions using different POD strategies. We also investigate the tracking performance of the international laser ranging network and show how highprecision orbits of LEO satellites can contribute to a better understanding and calibration of site-specific ranging errors. Sect. 2 starts with an overview of missions equipped with GPS receivers and Laser Retroreflector Arrays (LRAs), and discusses the present status of SLR-based orbit validation for satellites in low Earth orbit. Sect. 3 describes the methodology of SLR residuals computation and analysis, which is applied in Sect. 4 to investigate systematic errors in LEO orbits and laser ranging stations. Sect. 4.3 addresses laser ranging of formation flying satellites, which can be alternately tracked by the same station during a single pass. Finally, a summary is provided and conclusions drawn in Sect. 5.

\section{SLR for LEO Orbit Validation}

The increasing demand for high precision orbit products in geodetic and remote sensing missions in low Earth orbit (Roselló Guasch et al 2010; Quartly et al 2017) implies a need to assess the achievable orbit determination performance and to verify its compatibility with mission requirements. While observation residuals, internal consistency checks or cross-comparison to orbit solutions produced by independent software packages may provide some form of perfor- 
Table 1 Low Earth orbit satellite missions with GPS and SLR tracking

\begin{tabular}{|c|c|c|c|c|c|}
\hline Mission & Reference & Mission Period & Altitude $[\mathrm{km}]$ & GPS Receiver & LRA \\
\hline CHAMP & Reigber et al (2000) & $2000-2010$ & $450-180$ & BlackJack (JPL) & GFZ \\
\hline GRACE-A/B & Tapley et al (2004) & $2002-2017$ & $450-330$ & BlackJack (JPL) & GFZ \\
\hline Jason-1/2 & Lambin et al (2010) & 2001-2012 / since 2008 & 1300 & BlackJack (JPL) & ITE \\
\hline ICEsat & Schutz et al (2005) & 2003-2010 & 600 & BlackJack (JPL) & ITE \\
\hline GOCE & Floberghagen et al (2011) & 2009-2013 & $260-220$ & Lagrange (Laben) & IPIE \\
\hline TerraSAR-X/TanDEM-X & Buckreuss et al (2003); Krieger et al (2010) & Since $2007 / 2010$ & 515 & IGOR (BroadReach) & GFZ \\
\hline Swarm-A/B/C & Friis-Christensen et al (2008) & Since 2013 & $480 / 530 / 480$ & SGPS (RUAG) & GFZ \\
\hline Sentinel-3A & Fletcher (2012); Donlon et al (2012) & Since 2016 & 800 & SGPS (RUAG) & IPIE \\
\hline
\end{tabular}

mance metric and help to identify selected problems in a POD, a fully independent external orbit validation by means of other types of measurements is of key importance. Satellite laser ranging (Combrinck 2010) provides such a tool for independent orbit validation and has the advantage that the required on-board payload is merely a passive LRA.

Three basic LRA designs that are widely used in LEO missions are shown in Fig. 1. They comprise between four and nine individual prisms in a rotationally symmetric arrangement that ensures good reflection properties over a wide range of incidence angles. LRAs manufactured by the GeoForschungsZentrum (GFZ), Potsdam, have first been flown on the CHAMP and GRACE missions but are likewise used on the TerraSAR-X and TanDEM-X synthetic aperture radar (SAR) missions and the Swarm constellation. They consist of 4 corner cube prisms arranged in a regular $45^{\circ}$ pyramid (Neubert et al 1998). A seven-prism design was favored by the Russian Institute for Precision Instruments Engineering (IPIE; also known as Scientific Research Institute for Precision Instruments, or Research-and-Production Company for Precision Systems and Instruments), Moscow (Shargorodsky 2002). Reflector arrays made by IPIE are, for example flown on Cryosat, GOCE, PROBA-2, and Sentinel-3. Finally, a nine-prism retro-reflector array was designed by the United States' Instrumentation-Technology-Engineering (ITE) Inc. (later part of Honeywell Technology Solutions Inc., HTSI) and used onboard the ICEsat, ADEOS-II, and Jason-1/2/3 satellites ${ }^{1}$.

Table 1 presents an overview of LEO missions equipped with both a GPS receiver for POD and an LRA for satellite laser ranging. This list is not intended to be exhaustive but focuses on LEO missions considered in this study:

- The Challenging Minisatellite Payload (CHAMP, Reigber et al 2000) mission was used to study the Earth magnetic and gravity field and for atmospheric research. It was equipped with a $16 \times 3$-channel "BlackJack" (or Turbo Rogue Space Receiver, TRSR-2) GPS receiver manufactured by NASA's Jet Propulsion Laboratory (JPL). Up

\footnotetext{
1 https://ilrs.cddis.eosdis.nasa.gov/ missions/satellite_missions/current_missions/ jas2_reflector.html
}

to 10 satellites could be tracked for POD purposes using signals from a zenith-mounted choke ring antenna (Montenbruck and Kroes 2003). For SLR tracking a GFZ retroreflector array consisting of 4 corner cube prisms was employed. In the framework of the CHAMP Orbit Comparison Campaign Švehla and Rothacher (2003) validated reduced-dynamic and kinematic CHAMP orbits for 11 days in 2001. The authors reported root-meansquare (RMS) values of $4.4 \mathrm{~cm}$ for SLR residuals of reduceddynamic orbits and $5.6 \mathrm{~cm}$ for those of kinematic orbits, respectively.

- The Gravity Recovery And Climate Experiment (GRACE, Tapley et al 2004) was designed to measure the Earth's gravity field and especially its time variations with high spatial resolution. The mission consists of two satellites, GRACE-A and GRACE-B, orbiting the Earth on very similar orbits at a representative separation of $200 \mathrm{~km}$. GRACE uses a K-band inter-satellite link to measure the distance between both satellites with a precision of about $10 \mu \mathrm{m}$ with a $5 \mathrm{~s}$ data interval. The GRACE spacecraft are equipped with a similar type of GPS receiver (Dunn et al 2002) and the same LRA as CHAMP. A maximum of 10 GPS satellites were concurrently tracked for POD purposes. In their analysis of GPS-based orbit determination results for four months in the early mission phase, Kang et al (2006) report RMS SLR residuals of $2.5 \mathrm{~cm}$ for the two GRACE satellites. Slightly lower values of $1.8 \mathrm{~cm}$ were later obtained for POD solutions covering the full year of 2007 by Jäggi et al (2009).

- The Ice, Cloud, and land Elevation Satellite (ICEsat, Schutz et al 2005) carried the Geoscience Laser Altimeter System (GLAS) as its primary payload, which provided highprecision elevation data for determining the mass balance of the Greenland and Antarctic ice sheets. Precise orbits were derived from GPS tracking with a BlackJack GPS receiver (configured to track up to 8 satellites, Holt et al 2003) and partly using SLR. The 9-prism LRA uses the same design as the Jason satellites. To protect the GLAS instrument, no SLR tracking was permitted above $70^{\circ}$ elevation. Using observations from ten ILRS stations the University of Texas's Center for Space Research (UT/CSR) initially obtained RMS SLR residuals 
of $2.0 \mathrm{~cm}$. These could later be reduced to $1.4 \mathrm{~cm}$ on average over different mission phases through refined POD techniques (Yoon et al 2012).

- The Jason-1, -2, and -3 satellites (Lambin et al 2010) are part of a series of oceanography missions that started in 1992 with the US/French TOPEX/Poseidon mission. The primary payload of these missions consists of an altimeter that measures the satellite's height above the sea surface. Since radial orbit errors directly affect the derived sea level changes, orbit determination solutions of highest precision and accuracy are required for these missions. To achieve this goal, the Jason satellites are equipped with a DORIS (Doppler Orbitography and Radiopositioning Integrated by Satellite; Auriol and Tourain 2010) receiver, a BlackJack GPS receiver (Haines et al 2011) and a 9-prism laser retroreflector array. For Jason1 SLR residuals of $1.7 \mathrm{~cm}$ (RMS) were reported for 6 months of GPS-only reduced-dynamic orbit determination results in 2002 by Luthcke et al (2003). For comparison, daily mean RMS residuals of $2.0 \mathrm{~cm}$ (2002-2006) to $3.8 \mathrm{~cm}$ (2006-2009) were obtained for Jason-1 GPSonly orbit solutions in Flohrer et al (2011), while the corresponding value for Jason-2 amounts to $2.1 \mathrm{~cm}$ over the 2008-2009 time frame.

- The Gravity field and steady-state Ocean Circulation Explorer (GOCE; Floberghagen et al 2011) mission consisted of a single spacecraft equipped with a high-grade gradiometer for sensing the Earth's gravity field. To maintain the exceptionally low orbital altitude of $224-254 \mathrm{~km}$, the spacecraft made use of an electric propulsion system for drag compensation. GOCE used a 12×3-channel Lagrange GPS receiver (Zin et al 2006) for precise orbit determination and carried a 7-prism IPIE retroreflector specifically adapted to the low orbit height. In their analysis of precise science orbits (PSOs) generated by the GOCE high-level processing facility (HPF), Bock et al (2014) obtained SLR residuals of $1.8 \mathrm{~cm}$ for reduceddynamic orbits and $2.4 \mathrm{~cm}$ for kinematic orbits over the entire mission duration (RMS for April 2009 to Oct. 2013).

- TerraSAR-X and TanDEM-X satellites (Buckreuss et al 2003; Krieger et al 2010) are SAR satellites orbiting the Earth in a close formation to support bistatic interferometry and generation of a global digital elevation model. The spacecraft carry an Integrated Geodetic and Occultation Receiver (IGOR; Montenbruck et al 2006), which represents an industrialized version of the BlackJack receiver, and a complementary retroreflector of GFZ for orbit validation. Initial analyses of TerraSAR-X POD products resulted in SLR residuals of $2.2-2.9 \mathrm{~cm}$ (Yoon et al 2009), while RMS residuals of $1.7 \mathrm{~cm}$ and $1.3 \mathrm{~cm}$ where later reported in Hackel et al (2017) for the op- erational POD solutions and a new set of experimental products based on improved orbit models, respectively.

- The Swarm mission aims at a high-resolution spatial and temporal mapping of the Earth's magnetic field and the electric field of its atmosphere (Friis-Christensen et al 2008). It includes two formation flying satellites (SwarmA/C) at a typical separation of $100-150 \mathrm{~km}$ and a third spacecraft (Swarm-B) orbiting the Earth at a $50 \mathrm{~km}$ higher altitude and in a different orbital plane. All satellites are equipped with an $8 \times 3$-channel GPS receiver (Zangerl et al 2014) and the GFZ 4-prism retroreflector. The performance of the Swarm precise science orbits (PSOs) generated by the Delft University of Technology was assessed in van den IJssel et al (2015). Here, SLR residuals of $1.0-2.0 \mathrm{~cm}$ (RMS) were obtained for the individual satellites over a one year data arc in 2014, while kinematic orbits for this period exhibit RMS residuals of $4.1-4.7 \mathrm{~cm}$. Over a similar period, RMS residuals of $2.7-3.3 \mathrm{~cm}$ for the individual Swarm satellites were obtained by Jäggi et al (2016) using an independent set of kinematic orbits and high-quality SLR stations.

- The Sentinel-3 mission is part of the European Copernicus program and focuses on ocean monitoring (Fletcher 2012; Donlon et al 2012). Similar to Jason, high performance orbit determination is required for the processing of altimeter data, and the spacecraft is likewise equipped with a DORIS receiver, a GPS receiver (similar to that of Swarm, Montenbruck et al 2017b) and an SLR reflector (similar to that of GOCE). GPS-based precise orbit solutions are routinely generated as part of the Copernicus POD service (CPOD). SLR residuals of $1.8 \mathrm{~cm}$ (RMS) were obtained by Fernández et al (2016) in initial analyses of the CPOD orbit products for August 2016.

The aforementioned results evidence the relevance of SLR tracking for the validation of GPS-based precise orbit products and provide a first indication of the achievable POD performance. Unfortunately, published SLR residual statistics for the individual missions cannot be strictly compared due to the use of different sets of stations as well as different data screening limits applied by individual processing centers and authors.

An effort has therefore been made to analyze a set of publicly available orbit products for the individual LEOs in a consistent manner (Table 2). SLR observations for the analysis were provided by the International Laser Ranging Service (ILRS; Pearlman et al 2002). For each mission, we provide the SLR residuals statistics for both the full set of contributing stations as well as a subset of 12 high-quality stations (Graz, Greenbelt, Haleakala, Herstmonceux, Hartebeesthoek, Matera, Mt Stromlo, Potsdam, Yarragadee, Wettzell (two stations), and Zimmerwald). These high-quality stations typically contribute $50-75 \%$ of all tracking points and exhibit mean values and standard deviations of less than 
Table 2 Representative SLR residuals of LEO orbit products as determined in the present study from various public and contributed orbit products using a consistent set of stations and data screening thresholds.

\begin{tabular}{llccc}
\hline Mission & Orbit product & Year & \multicolumn{2}{c}{ Residuals (mean $\pm \sigma$ [mm]) } \\
& & & All stations & High-quality stations \\
\hline CHAMP & AIUB red. dyn. (Prange et al 2010) & 2007 & $+2.6 \pm 23.0$ & $+1.5 \pm 18.2$ \\
& AIUB kinematic (Prange et al 2010) & 2007 & $+0.6 \pm 34.4$ & $+0.8 \pm 31.4$ \\
GRACE-A & JPL GNV1B (Bettadpur 2012; Bertiger et al 2010) & 2010 & $+2.3 \pm 24.4$ & $+3.1 \pm 12.3$ \\
ICEsat & UT/CSR 2011 reprocessing (Rim et al 2013) & 2008 & $+2.4 \pm 15.4$ & $+2.0 \pm 15.2$ \\
Jason-2 & CNES GPS+DORIS GDR-E (CNES 2015; IDS 2015) & 2016 & $-6.1 \pm 25.3$ & $+0.6 \pm 12.5$ \\
GOCE & AIUB PSO red. dyn. (Bock et al 2014) & 2010 & $+2.6 \pm 21.0$ & $+2.6 \pm 13.8$ \\
& AIUB PSO kinematic (Bock et al 2014) & 2010 & $+2.7 \pm 23.3$ & $+2.9 \pm 17.1$ \\
TerraSAR-X X & DLR red. dyn. (Hackel et al 2017) & 2016 & $+3.5 \pm 25.4$ & $+3.4 \pm 15.3$ \\
Swarm-B & TU Delft PSO red. dyn. (van den IJssel et al 2015) & 2016 & $+0.3 \pm 25.5$ & $+0.3 \pm 15.2$ \\
& TU Delft PSO kinematic (van den IJssel et al 2015) & 2016 & $+0.7 \pm 31.2$ & $+0.8 \pm 24.3$ \\
Sentinel-3A & CPOD (Peter et al 2016) & 2016 & $+1.8 \pm 27.2$ & $+2.6 \pm 15.7$ \\
\hline
\end{tabular}

$20 \mathrm{~mm}$ for a large range of missions, which distinguishes them from most of the other stations. Even though their choice is not necessarily complete and partly arbitrary, it clearly helps to discriminate different performance levels among the stations of the ILRS network.

A $10^{\circ}$ elevation mask and a $20 \mathrm{~cm}$ threshold for outlier rejection were applied in all cases. Line-of-sight dependent range corrections were applied taking into account the properties of the mission-specific LRAs. Further details of the residual computation are addressed in Sect. 3 below.

The results given in Table 2 demonstrate a $1.5-2.5 \mathrm{~cm}$ consistency of GPS-based reduced-dynamic orbit solutions with the SLR measurements. Slightly inferior results are also achieved for kinematic orbit products that do not benefit from the constraints of a dynamical orbit model.

By their very nature, SLR residuals constitute a measure of the 1-dimensional (1D) orbit error. Under the simplifying assumption of zero mean errors and identical standard deviations in all directions, a rule-of-thumb estimate of the corresponding $3 \mathrm{D}$ position error is given by $\sigma_{\mathrm{pos}}=\sqrt{3} \sigma_{\mathrm{SLR}}$, which amounts to $2.5-4.5 \mathrm{~cm}$ for the aforementioned orbit products. A similar relation $\sigma_{\mathrm{pos}}=(1.5 \pm 0.2) \sigma_{\mathrm{SLR}}$ between orbit errors and SLR residuals was established empirically by Boomkamp (2003) in the frame of the CHAMP orbit comparison campaign, but might need modification for different missions and orbital altitudes.

In practice, however, SLR residuals of LEO satellite provide an average of the projected position error over a limit range of line-of-sight directions. Depending on the satellite's altitude and the station elevation mask, the lines-ofsight are confined to a cone of about $50-80^{\circ}$ about the satellite's nadir direction. The SLR measurements are therefore most sensitive to radial contributions of the orbit error, which is more tightly constrained than the along-track component in a reduced-dynamic orbit determination. As such, SLR residual analyses may underestimate the overall position error if the observation geometry is not well taken into consideration.
On the other hand, SLR residuals are not a measure of orbit errors alone, but include additional error contributions related to the laser ranging measurement and its modeling. A comparison of SLR residuals for different sets of stations in Table 2 clearly shows that these errors may indeed constitute a limiting factor for the quality assessment of highperformance orbit products. This is best seen for Jason-2 that shows a factor of two improvement in the RMS residuals, when considering only measurements from stations known for their good data quality. While the histogram of SLR residuals over all stations exhibits notable distortions (Fig. 2), a much narrower and near-Gaussian distribution is obtained for the aforementioned selection of high-performance stations. Obviously, due care must be taken to ensure that SLR sensor noise and calibrations as well as measurement models used in the data analysis are fully competitive with the accuracy of advanced orbit products for present LEO missions. Before investigating these aspects in more detail, we provide a summary of state-of-the-art SLR models and present specific processing strategies that can help to identify systematic measurement or orbit errors.

\section{Methods}

Satellite laser ranging stations measure the turn-around time of a signal emitted by the station and received again after being reflected at the target satellite. The total ("2-way") time of flight $\tau$ given by the timing measurement can, alternatively, be expressed as a range value

$\rho=\frac{1}{2} \cdot c \tau$,

where $c$ denotes the vacuum speed of light. This range is essentially the "1-way" distance between the target satellite and the SLR station, but various signal propagation effects must be taken into account for a more rigorous modeling. Throughout this publication, "SLR residuals" refer to the difference $\rho_{\mathrm{o}}-\rho_{\mathrm{c}}$ of observed and computed range values 

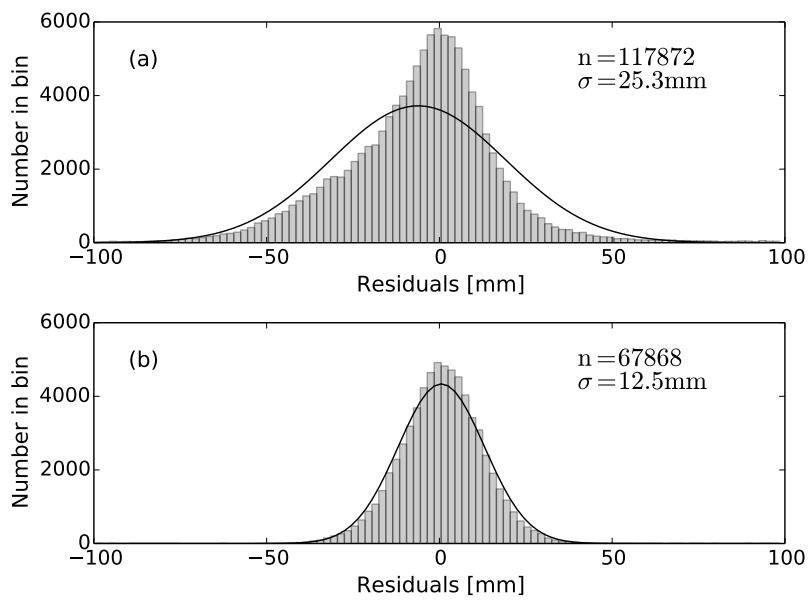

Fig. 2 Histogram of SLR residuals for GDR-E Jason-2 orbits in 2016 considering data from all ILRS stations (a) and considering only a subset of high-grade stations (b). In addition, the total number of SLR observations and the standard deviation of the residuals are given. The solid lines describe a normal distribution with the corresponding standard deviation

based on Eqn. (1). An overview of key contributions considered in the SLR range model is given in Table 3.

Table 3 Models for processing of satellite laser ranging data from LEO satellites

\begin{tabular}{|c|c|}
\hline Component & Description \\
\hline Station coordinates & $\begin{array}{l}\text { SLRF2008 (v16/08/08) (ILRS Analy- } \\
\text { sis Standing Committee 2016) } \\
\text { SLRF2014 (v17/06/05) (ILRS 2017) } \\
\text { and ITRF2014 postseismic deforma- } \\
\text { tions (ITRF 2017) }\end{array}$ \\
\hline Solid Earth and pole tides & IERS2010 (Petit and Luzum 2010) \\
\hline Ocean tide loading & FES2004 (Lyard et al 2006) \\
\hline Atmospheric pressure loading & $\begin{array}{l}\text { Wijaya et al (2013); GGOSATM } \\
\text { (2017) (optional; see text) }\end{array}$ \\
\hline Satellite coordinates & CoM trajectory from GPS-based POD \\
\hline Center-of-mass position & From satellite telemetry \\
\hline LRA reference point position & From satellite design \\
\hline & From star camera quaternio \\
\hline & IERS2010 (Mendes and Pavlis 2004) \\
\hline Relativity & $\begin{array}{l}\text { Space-time curvature correction (Petit } \\
\text { and Luzum 2010) }\end{array}$ \\
\hline LRA range correction & $\begin{array}{l}\text { LRA specific (Neubert 2009; Mon- } \\
\text { tenbruck and Neubert 2011) }\end{array}$ \\
\hline
\end{tabular}

\subsection{Range Model}

Given the ground receive time $t$ of the return signal as measured by the station and reported in the consolidated laser ranging data format (CRD; Ricklefs and Moore 2008), a range measurement may be described by the model

$\rho=\frac{1}{2}\left(\rho_{\text {up }}(t)+\rho_{\text {down }}(t)\right)+\Delta \rho_{\text {rel }}+\Delta \rho_{\text {trop }}+\Delta \rho_{\text {LRA }}+\epsilon$, where $\rho_{\text {up }}$ and $\rho_{\text {down }}$ are the (light-time corrected) up- and downlink distances between the reference points of the station and the satellite. The relativistic correction $\Delta \rho_{\text {rel }}$ takes into account the range delay caused by the gravitational deflection of light in the vicinity of the Earth, $\Delta \rho_{\text {trop }}$ is the tropospheric range delay and $\Delta \rho_{\mathrm{LRA}}$ is an LRA-specific range correction that takes into account the difference between the station distance from the LRA reference point and the actual signal path within the LRA (see Sect. 3.2). $\epsilon$ contains satellite- and station-specific systematic errors which affect the measured range, in particular orbit offsets (see Sect. 4.1), range biases $b$ (see Sect. 4.2.1), and range errors $\Delta \rho_{t}$ introduced by timing offsets at the stations (see Sect. 4.2.3). In accordance with Eqn. (1) all corrections refer to a single leg of the total signal path, or, more rigorously, the arithmetic average of the up- and downlink.

When working in an inertial reference frame, the up- and downlink ranges are described by the equations

$$
\begin{aligned}
& \rho_{\text {up }}(t)=\left\|\boldsymbol{r}\left(t-\tau_{\text {down }}\right)-\boldsymbol{R}\left(t-\tau_{\text {down }}-\tau_{\text {up }}\right)\right\| \\
& \rho_{\text {down }}(t)=\left\|\boldsymbol{r}\left(t-\tau_{\text {down }}\right)-\boldsymbol{R}(t)\right\|,
\end{aligned}
$$

where $\boldsymbol{r}$ and $\boldsymbol{R}$ denote the time-dependent positions of the LEO satellite and the SLR station, respectively. The unknown up- and downlink light times are implicitly defined by the relations

$$
\begin{aligned}
& \tau_{\text {up }}=\left\|\boldsymbol{r}\left(t-\tau_{\text {down }}\right)-\boldsymbol{R}\left(t-\tau_{\text {down }}-\tau_{\text {up }}\right)\right\| / c \\
& \tau_{\text {down }}=\left\|\boldsymbol{r}\left(t-\tau_{\text {down }}\right)-\boldsymbol{R}(t)\right\| / c
\end{aligned}
$$

and can be iteratively solved using initial approximations

$\tau_{\text {up }}^{(0)}=\tau_{\text {down }}^{(0)}=\|\boldsymbol{r}(t)-\boldsymbol{R}(t)\| / c$.

Expanding the motion of the station around the instant of signal reflection at the satellite, neglecting quadratic terms in the total light time $\tau=\tau_{\text {up }}+\tau_{\text {down }}$, and assuming $\tau_{\text {up }} \approx$ $\tau_{\text {down }} \approx \tau / 2$, the simplified relation

$\frac{1}{2}\left(\rho_{\text {up }}(t)+\rho_{\text {down }}(t)\right) \approx\|\boldsymbol{r}(t-\tau / 2)-\boldsymbol{R}(t-\tau / 2)\|$

is obtained, which provides an adequate approximation for most purposes. For LEO satellites the approximation error is below $0.5 \mathrm{~mm}$.

Given the fact that both station coordinates and GPSbased LEO satellite orbits are most commonly expressed in an Earth-fixed (ef) reference frame, an alternate formulation

$$
\begin{gathered}
\rho_{\text {up }}(t)=\| \boldsymbol{r}^{\mathrm{ef}}\left(t-\tau_{\text {down }}\right) \\
-\boldsymbol{R}_{z}\left(-\omega_{\oplus} \tau_{\text {up }}\right) \boldsymbol{R}^{\mathrm{ef}}\left(t-\tau_{\text {down }}-\tau_{\text {up }}\right) \| \\
\rho_{\text {down }}(t)=\| \boldsymbol{r}^{\mathrm{ef}}\left(t-\tau_{\text {down }}\right) \\
-\boldsymbol{R}_{z}\left(+\omega_{\oplus} \tau_{\text {down }}\right) \boldsymbol{R}^{\mathrm{ef}}(t) \|,
\end{gathered}
$$


of Eqn. (3) (and equivalently Eqn. (4)) is useful that takes into account the Earth rotation and avoids the explicit transformation of station and satellite coordinates into an inertial frame. Here, $\boldsymbol{R}_{z}\left(\omega_{\oplus} \tau\right)$ describes the differential Earth rotation over a small time interval $\tau$ assuming a pure $z$-axis rotation at the Earth's angular velocity $\omega_{\oplus}$. The corresponding terms in Eqn. (7) ensure that the station-satellite position difference is formed in a common (non-rotating) frame with the Earth's axes at the instant $t-\tau_{\text {down }}$ of signal reflection at the LEO satellite. They are also known as Sagnac correction and arise when describing the propagation of light signals in a rotating reference frame both in a Newtonian and relativistic formulation (Ashby 2004). For SLR tracking of LEO satellites, peak values of the correction for either the up- or downlink range from $2 \mathrm{~m}(7 \mathrm{~ns})$ at $500 \mathrm{~km}$ altitude to twice that value at $1300 \mathrm{~km}$. Since both contributions are of opposite sign, the combined effect on the modeled turn-around range cancels at the level of $0.1 \mathrm{~mm}$.

SLR station coordinates are provided as part of the SLR contribution (e.g., SLRF2008 or SLRF2014) to the International Terrestrial Reference Frame (ITRF). The respective positions are extrapolated to the time of interest using the site velocity as well as potential post-seismic deformation parameters (since SLRF2014; see Altamimi et al 2016). In addition, time-dependent tidal motions are considered based on conventional solid Earth, ocean and pole tide models (Petit and Luzum 2010) and site specific ocean loading coefficients made available through the Onsala Space Observatory (Chalmers OSO 2016). Further refinement of the station coordinate modeling can be achieved by consideration of atmospheric pressure loading (APL), which may induce position changes at the few-mm to one-cm level (Bury and Sośnica 2017). For the assessment of APL effects on the analysis of LEO SLR data (see Sect. 4.2) we make use of 6-hourly APL values provided by the Technical University of Vienna on a $1^{\circ} \times 1^{\circ}$ longitude/latitude grid (Wijaya et al 2013).

The motion of the LRA reference point onboard the LEO satellite is obtained by adding the offset vector of the LRA with respect to the center of mass (CoM) to the CoM trajectory as interpolated from the LEO POD product. To obtain this offset vector in the analysis reference frame knowledge of the spacecraft orientation is required. In view of the high precision SLR observations, attitude quaternions describing the true (rather than nominal) orientation of the spacecraft body frame must be made available through the spacecraft operator along with information on the CoM and LRA position in this frame.

Strictly speaking, use of Eqns. (3) and (7) implies that station and satellite coordinates are referred to an identical reference frame, but this requirement cannot be rigorously met in practice. Through the GPS orbit products used in the POD processing, LEO satellite orbits derived from GPS observations are implicitly tied to the International GNSS Service (IGS, Dow et al 2009) realization of the International Terrestrial Reference System. SLR site coordinates, on the other hand, refer to an SLR-specific realization. It is generally assumed that the associated differences can be neglected when working with corresponding frame realizations, i.e., the technique-dependent frames contributing to a common release of the International Terrestrial Reference Frame (e.g., IGS08/IGb08 (Rebischung et al 2012; Rebischung 2012) and SLRF2008 (ILRS Analysis Standing Committee 2016) for ITRF2008).

On top of the basic free-space signal propagation model of Eqns. (3)/(7) a general relativistic correction

$$
\Delta \rho_{\mathrm{rel}}=\frac{2 G M_{\oplus}}{c^{2}} \ln \left(\frac{\|\boldsymbol{r}\|+\|\boldsymbol{R}\|+\rho}{\|\boldsymbol{r}\|+\|\boldsymbol{R}\|-\rho}\right),
$$

is required to take into account the range change caused by gravitational bending of the light path (Shapiro effect; Petit and Luzum 2010). The ratio $2 G M_{\oplus} / c^{2}$ of twice the Earth's gravitational coefficient and the squared speed of light denotes the Schwarzschild radius of the Earth and amounts to roughly $9 \mathrm{~mm}$. For LEO satellites with orbital altitudes of $200-2000 \mathrm{~km}$ ) the resulting effect on the SLR measurement varies between about $0.3-2.5 \mathrm{~mm}$ at zenith and $2-7 \mathrm{~mm}$ at the horizon.

Finally, tropospheric range delays need to be taken into account in the SLR range model. In accordance with current IERS conventions (Petit and Luzum 2010), the zenith delay and mapping function for optical wavelength of Mendes and Pavlis (2004) are employed along with meteorological data reported by the SLR stations along with the ranging observations. The tropospheric delay ranges from about $2 \mathrm{~m}$ at zenith to $14 \mathrm{~m}$ at $10^{\circ}$ elevation and can be modeled with an accuracy of about $7 \mathrm{~mm}$ (combined error of zenith delay and mapping function; Petit and Luzum 2010, p. 11).

\subsection{LRA Range Correction}

The up- and downlink ranges modeled by Eqns. (3) and (7) refer to a mission-specific LRA reference point. This may either be a mechanical reference point (MRP; e.g., the center of the mounting plane) or an optical reference point (ORP; e.g., intersection of the optical axes of all prisms). The actual SLR range differs from the modeled range of the reference point for two reasons. First, part of the light path takes place inside the prism, where the propagation speed is lower than in vacuum. Secondly, the distance from the station to the individual prisms differs from reference point distance by the line-of-sight projection of the prisms offset from the reference point. Following Neubert et al (1998), the range correction for a single prism $i$ can be described by

$\Delta \rho_{\mathrm{LRA}, i}=\left[L \sqrt{n_{\mathrm{g}}^{2}+\left(\boldsymbol{e}^{T} \boldsymbol{n}_{i}\right)^{2}-1}\right]-\left[\boldsymbol{e}^{T} \boldsymbol{r}_{i}\right]$, 

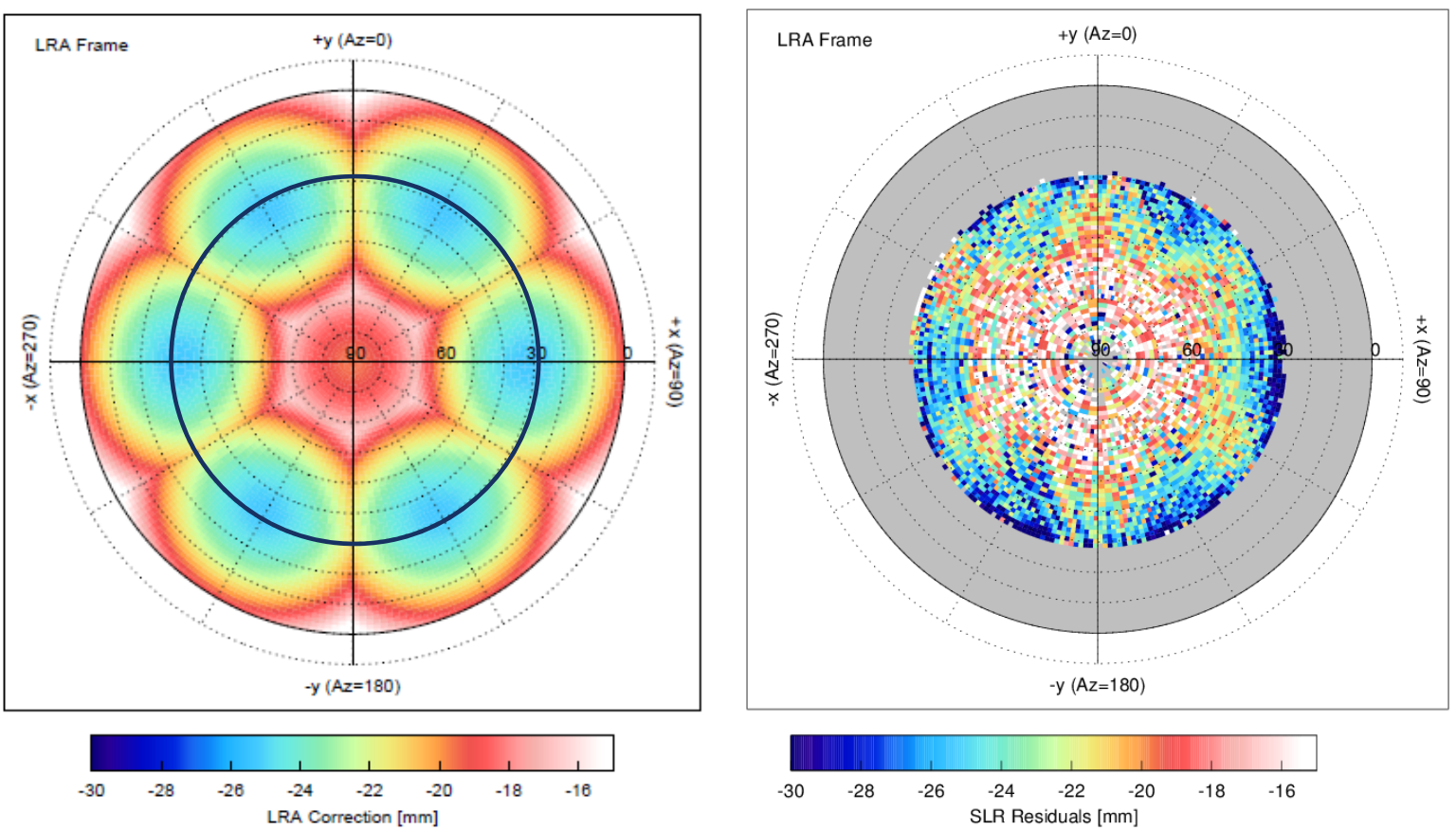

Fig. 3 Range correction for Sentinel-3 (nearest prism approximation; left) and comparison with SLR residuals obtained in absence of the range correction (right). The polar diagrams show the color coded value of the range correction (or residual) as a function of the azimuth and elevation of the line-of-sight direction in the LRA reference system. The region encircled by the bold black line in the left subplot marks the line-of-sight directions corresponding to station elevations above $10^{\circ}$

where the two terms in square brackets describe the contributions of the internal light path and the prism offset, respectively. Here $e$ denotes the line-of-sight unit vector from the LRA to the station, $\boldsymbol{r}_{i}$ is the offset of the prism's input face center from the reference point, and $\boldsymbol{n}_{i}$ is the unit vector along the optical axis of the prism. Furthermore, $L$ and $n_{\mathrm{g}}$ are the vertex height of the prism (i.e., the distance between the input face plane and the prism vertex) and its group refractive index.

Depending on the signal strength and detector system of the SLR station, the total range correction for a multi-prism LRA is either determined by the nearest prism or a weighted average of prisms with an optical axis sufficiently close to the line-of-sight direction (Neubert et al 1998). While the multi-prism yields a fairly smooth range correction, steeper variations in the transition region between the fields-of-view of adjacent prisms may be observed in the nearest-prism range correction model, which is suggested for single-photon systems (R. Neubert, priv. comm.).

Range corrections over a two dimensional grid of direction angles were derived in Neubert (2009) for the 4-prism LRA of GFZ and in Montenbruck and Neubert (2011) for the two types of IPIE 7-prism retroreflector arrays.

A comparison of the modeled LRA range correction with range residuals obtained in absence of the correction is shown in Fig. 3 for 10 months of Sentinel-3A laser ranging observations. The range correction pattern is clearly visible in the residuals and omission of the LRA correction introduces a pronounced bias of about $-23 \mathrm{~mm}$. Superimposed azimuthand elevation-dependent variations amount to roughly $\pm 5 \mathrm{~mm}$ (peak-to-peak) for this specific LRA design. Consideration of the line-of-sight dependent correction results in a small, but still relevant, decrease of the standard deviation of the residuals as compared to a neglected or constant range correction. This is most evident for high-performance stations where a reduction at the $0.5 \mathrm{~mm}$ level is obtained.

\subsection{Bias and Offset Estimation}

While the performance assessment of GPS-based orbit determination using SLR observations is most commonly based on simple residuals statistics (e.g., mean, standard deviation, and/or RMS), further insight can be gained from the variation of residuals throughout a pass. As discussed in, e.g., Combrinck (2010) and McWilliams (2015), range and timing biases as well as station position and orbit offsets yield distinct variations in the range observations, which can in turn be used to infer corrections to the respective parameters from the residuals analysis. By way of example, a stationspecific ranging bias will show up in a non-zero mean value of the residuals for that station, while a station timing bias will results in SLR residuals proportional to the range rate (or Doppler shift) across each pass.

Given a set of SLR observations $\rho_{\mathrm{o}, i}, i=1, \ldots, n_{\mathrm{obs}}$, a set of parameters of interest $p_{k}, k=1, \ldots, n_{\mathrm{p}}$ can be 
determined such that the computed ranges $\rho_{\mathrm{c}, i}\left(p_{k}\right)$ best fit the observations

$\rho_{\mathrm{o}, i}=\rho_{\mathrm{c}, i}\left(p_{k}\right)+v_{i}$

in a least-squares sense, where $v_{i}$ are the observation residuals to be minimized. Linearization around $p_{k}=0$ yields the equations

$r_{i}=\sum_{k=1}^{n_{\mathrm{p}}} \frac{\partial \rho_{\mathrm{c}, i}}{\partial p_{k}} p_{k}+v_{i}$,

where $r_{i}=\rho_{\mathrm{o}, i}-\rho_{\mathrm{c}, i}(0)$ are the classical SLR residuals obtained without parameter estimation, and where the partial derivatives are evaluated at $p_{k}=0$.

Other than a direct incorporation of SLR observations and the respective estimation parameters into a GPS+SLRbased orbit determination, the residual processing is well suited to study slowly varying biases and offsets that are difficult to estimate in short (daily) processing arcs. Also, residual-based parameter analyses can be performed based on published orbit solutions without requiring access to the full set of associated normal equations.

The required partial derivatives depend on the specific choice of parameters under study, but can conveniently be obtained as a byproduct of the range modeling itself. Regarding LEO position errors, a set of constant orbit offsets $\Delta r_{\mathrm{R}}, \Delta r_{\mathrm{T}}$, and $\Delta r_{\mathrm{N}}$, in radial (R), along-track (T), and normal $(\mathrm{N})$ direction may be considered to investigate systematic force modeling errors, while constant offsets $\Delta r_{\mathrm{x}}, \Delta r_{\mathrm{y}}$, and $\Delta r_{\mathrm{z}}$ along the spacecraft body axes may be preferred for the assessment of equipment accommodation offsets. Considering only the geometric part in the computed range, the respective partials

$\frac{\partial \rho_{\mathrm{c}, i}}{\partial\left(\Delta r_{\mathrm{R}}, \Delta r_{\mathrm{T}}, \Delta r_{\mathrm{N}}\right)^{T}}=\left(\boldsymbol{e}^{T} \boldsymbol{e}_{\mathrm{R}}, \boldsymbol{e}^{T} \boldsymbol{e}_{\mathrm{T}}, \boldsymbol{e}^{T} \boldsymbol{e}_{\mathrm{N}}\right)_{i}$

and

$\frac{\partial \rho_{\mathrm{c}, i}}{\partial\left(\Delta r_{\mathrm{x}}, \Delta r_{\mathrm{y}}, \Delta r_{\mathrm{z}}\right)^{T}}=\left(\boldsymbol{e}^{T} \boldsymbol{e}_{\mathrm{x}}, \boldsymbol{e}^{T} \boldsymbol{e}_{\mathrm{y}}, \boldsymbol{e}^{T} \boldsymbol{e}_{\mathrm{z}}\right)_{i}$

are obtained by projection of the RTN or $x y z$ unit vectors on the line-of-sight-unit vector $\boldsymbol{e}=(\boldsymbol{r}-\boldsymbol{R}) /\|\boldsymbol{r}-\boldsymbol{R}\|$. Notice that the right hand sides are simply the components of the line-of-sight unit vector in the RTN or $x y z$ coordinate frame, respectively. In a similar way, partials of the modeled range with respect to station coordinate offsets in east (e), north (n), and up (u) direction are given by

$\frac{\partial \rho_{\mathrm{c}, i}}{\partial\left(\Delta R_{\mathrm{e}}, \Delta R_{\mathrm{n}}, \Delta R_{\mathrm{u}}\right)^{T}}=-\left(\boldsymbol{e}^{T} \boldsymbol{e}_{\mathrm{e}}, \boldsymbol{e}^{T} \boldsymbol{e}_{\mathrm{n}}, \boldsymbol{e}^{T} \boldsymbol{e}_{\mathrm{u}}\right)_{i}$.

Range biases $b$ are treated as additive constants in the modeled range and therefore result in unit partial derivatives

$\frac{\partial \rho_{\mathrm{c}, i}}{\partial b}=1$ for the respective tracking station. Finally, the impact of station-specific timing offsets $\Delta t$ is described by the partial derivative

$$
\frac{\partial \rho_{\mathrm{c}, i}}{\partial \Delta t}=\left(\boldsymbol{e}^{T}(\boldsymbol{v}-\boldsymbol{V})\right)_{i}
$$

where $\boldsymbol{v}$ and $\boldsymbol{V}$ are the inertial velocity vectors of the satellite and the station.

As described in McWilliams (2015), station and orbit offsets may result in (almost) identical variations of the range residuals across a single tracking pass, and are only weakly decorrelated as a result of the Earth's rotation. Likewise, a global timing error is indistinguishable from a systematic orbit offset in along-track direction. The parameter estimation is therefore best performed using long data arcs (depending on the time scales to be resolved typically one month to one year), multiple stations and, optionally, multiple satellites along with suitable a priori constraints for individual parameters or parameter combinations.

Applications of the residuals-based parameter estimation are, e.g., discussed in Hackel et al (2017) who evidenced seasonally varying cross-track offsets in LEO orbits based on low-grade non-gravitational force models, and in Montenbruck et al (2017b), who inferred a systematic center-of-mass offset for the Sentinel-3A satellite.

\section{SLR Quality Assessment and Error Analysis}

Beyond the obvious use of SLR residuals statistics as a quality criterion for LEO orbits, the parameter estimation approach presented in Sect. 3.3 can be used to gain further insight into systematic errors of individual orbit products and the employed laser ranging network. These aspects are separately discussed in Sects. 4.1 and 4.2, respectively.

\subsection{Orbit Errors}

To illustrate the analysis of systematic orbit errors in GPSbased LEO orbit products, we first present an analysis of Sentinel-3A orbit solutions obtained with and without phase pattern corrections. For this analysis reduced-dynamic Sentinel3A orbits were computed by the Astronomical Institute of the University of Bern (AIUB), covering the days of year (DOY) 054-335 of 2016. Initial orbit solutions were computed with nominal phase center offset information for this satellite. Using a residual stacking approach (Jäggi et al 2009), phase variation (PV) maps of the main POD GPS antenna were estimated and applied.

Comparison of consecutive orbit solutions evidences systematic cross-track offsets between individual solutions (see Fig. 4), but this does not allow to discriminate which of 
these solutions best represents the true trajectory. For all orbit solutions radial, along-track, and cross-track offsets have therefore been estimated from SLR observations using the aforementioned high-quality stations. The cross-track values are shown in Figure 4 as well. Both the mean orbit differences and the offset estimation are based on the period covering DOY 092-335, since Sentinel-3A was tracked by SLR only starting on April 1st, 2016 (DOY 092).

It can be seen that the orbit solution obtained without PV correction exhibits a cross-track offset of nearly $2 \mathrm{~cm}$. While this value decreases to almost zero after four iterations, further iterations increase it again and it converges towards a non-zero offset of approximately $5 \mathrm{~mm}$. The SLR-derived offsets for orbits from different iterations (green curve in Fig. 4) are consistent with the respective orbit differences (red curve in Fig. 4) at the $0.3 \mathrm{~mm}$ level. While it is frequently assumed that SLR residuals are indicators of only the radial orbit errors, the present results illustrate that SLR tracking of LEO satellites can also be used with good confidence to assess systematic orbit errors in the perpendicular (i.e., cross-track and along-track) direction. It must be noted, though, that the interpretation of the resulting offsets is in no way unambiguous and may be affected by improper information on the LRA-CoM offset.

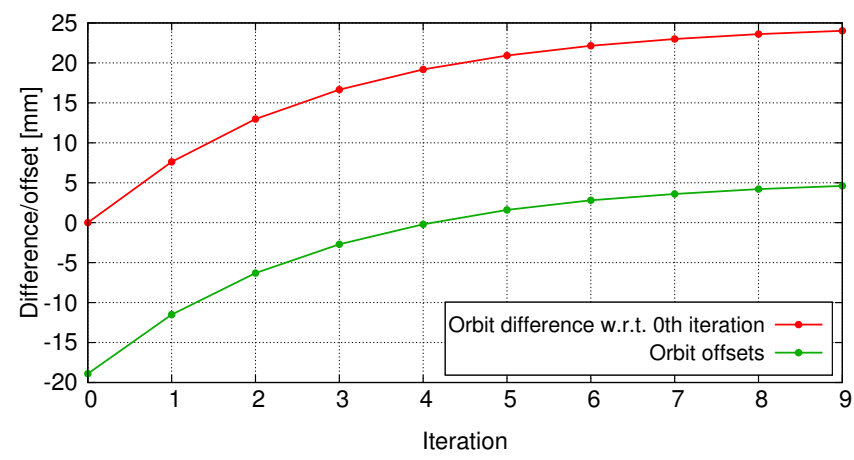

Fig. 4 Red: Mean differences in normal direction of orbit solutions obtained in iterations of a Sentinel-3A PV map generation w.r.t. the orbit solution obtained without applying PV corrections (iteration 0). Green: Orbit offset in normal direction estimated from SLR observations of high-quality stations over the period April to November 2016. SLRF2008 site coordinates were used.

As a second example, we compare three different sets of Sentinel-3 orbit solutions computed by the AIUB, the Copernicus POD service (CPOD), and the German Aerospace Center (DLR). The three solutions mainly differ in their choice of float vs fixed ambiguity and the handling of non-gravitational forces. To assess any systematic differences between the solutions, position offsets relative to the orbital frame were derived from SLR tracking over a nine months period (Table 4).

Aside from an overall reduction of the SLR residuals, the ambiguity-fixed solution is almost free of cross-track bi- ases, while systematic offsets perpendicular to the flight direction may be inferred for the float-ambiguity solutions. As discussed in Montenbruck et al (2017b), the bias can likely be attributed to erroneous information on the lateral position of the center of mass (CoM), whereas the SLR-GPS offset is correctly reflected by spacecraft design data. In the float-ambiguity processing, the dynamical modeling aligns the modeled CoM with the true orbit and absorbs the incorrect lateral offset of the GPS antenna in the estimated ambiguities. The ambiguity-fixed solution, in contrast, properly represents the trajectory of the GPS antenna and LRA relative to the orbital plane, but results in a lateral shift of the CoM, which evidences itself through significant empirical accelerations in cross-track direction.

In radial direction, the estimated CoM trajectory (and thus also the corresponding LRA trajectory) is mainly governed by the dynamical models. A consistent radial leveling may be observed for the two processing schemes using macro models for the modeling of non-gravitational forces. The AIUB processing results, in contrast show a different radial offset due to the kinematic leveling (by estimation of a free radial acceleration), which relies on correct antenna/CoM information (see Peter et al (2017) for a more detailed discussion of this aspect). It must be stated, though, that SLR tracking cannot serve as an absolute truth reference for the assessment of radial orbit errors, since the results depend itself on a couple of other errors sources. Aside from uncertainties in the knowledge of the LRA-CoM offset vector and the LRA range corrections provided by the spacecraft operator or manufacturer, the SLR-derived radial position offsets are highly affected by station specific range biases or height offsets that will be further addressed in the subsequent section. Among others, this correlation shows up in a $4 \mathrm{~mm}$ shift of the derived radial offset when switching from SLRF2008 to SLRF2014 station coordinates (Table 4).

\subsection{Station Errors}

For the analysis of ILRS network characteristics, we make use of a dedicated set of GPS-based precise orbit determination solutions for a total of four LEO missions (Swarm-C, TerraSAR-X, Sentinel-3A, and Jason-2) covering an altitude range of about $500-1300 \mathrm{~km}$. Most of these orbit products were prepared specifically for this study using a reduceddynamic approach with macro models for the non-gravitational forces and single-receiver ambiguity fixing. Details of the employed models and processing standards are described in Montenbruck et al (2017b). Compared to non-ambiguity fixed solutions (such as those discussed before in Sect. 2), the present products offer a notably enhanced precision and are therefore best suited to assess the tracking network performance and individual station characteristics. 
Table 4 Orbit offsets in radial (R), along-track (T) and normal (N) direction, and RMS residuals (Res) of three Sentinel-3A POD solutions based on SLR tracking of high-quality stations over the period from April to December 2016. Independent results are provided for use of SLRF2008 and SLRF2014 site coordinates. All values in [mm]

\begin{tabular}{|c|c|c|c|c|c|c|c|c|c|}
\hline \multirow[t]{2}{*}{ Solution } & \multicolumn{4}{|c|}{ SLRF2008 } & \multicolumn{4}{|c|}{ SLRF2014 } & \multirow[t]{2}{*}{ Notes } \\
\hline & $\mathrm{R}$ & $\mathrm{T}$ & $\mathrm{N}$ & Res & $\mathrm{R}$ & $\mathrm{T}$ & $\mathrm{N}$ & Res & \\
\hline AIUB & -1.5 & -3.9 & +4.6 & 13.6 & -5.5 & -3.3 & +5.0 & 12.4 & Float ambiguity, free accel. in RTN \\
\hline CPOD & +4.7 & -7.1 & +8.5 & 14.5 & +0.8 & -6.5 & +8.9 & 13.8 & Float ambiguity, macro model \\
\hline DLR & +4.7 & -0.4 & +0.7 & 11.5 & +0.7 & +0.2 & +1.1 & 10.6 & Ambiguity-fixed, macro model \\
\hline
\end{tabular}

With the exception of Sentinel-3A that only became available in late March 2016, the data set covers the entire year of 2016 during which the four spacecraft were tracked by a total of 37 ILRS stations. Out of these, five stations (Altay, Kiev, Komsomolsk, San Fernando, and Simeiz) were discarded in our analysis because of extremely sparse tracking or unusually large tracking residuals. Overall, some 230000 normal points were collected for the four satellites by the selected set of ILRS stations over the one-year interval.

Aside from Jason with an orbital inclination of $66^{\circ}$, all missions considered in the analysis use polar orbits and are usually tracked in ascending (south-to-north) and descending (north-to-south) passes. Possible errors in the horizontal station positions can thus be separated from in-plane position errors of the satellite orbit and show up in a systematic azimuth dependence of the residuals. Height errors, in contrast, can be inferred from a $\sin (E)$-dependence of the residuals on elevation $E$. When estimating station height errors, it is, furthermore, mandatory to also adjust the range bias. Otherwise, height corrections would remove the elevation dependence but might increase the overall sum of the squared residuals.

\subsubsection{Station positions and range biases}

Based on these considerations, corrections to SLRF2008 a priori positions and range biases were adjusted for all stations as described in Sect. 3.3. The need to adjust the range biases along with the station coordinates was earlier emphasized by Coulot et al (2007). No simultaneous orbit adjustments were considered, since potential horizontal orbit errors will average out over the mix of ascending and descending passes, while estimation of orbit and station heights is fully correlated. As such, the resulting station height corrections are specific to the employed orbit products and subject to change when using other LEO missions or product types. Possible systematic biases in the adopted CoM-LRA offsets or mismodeled radial accelerations (see Sect. 4.1) will directly affect the inferred station height in the analysis.

Depending on the coverage of individual stations, formal uncertainties of about $1-2 \mathrm{~mm}$ and $2-4 \mathrm{~mm}$ are obtained for the horizontal and vertical components of the position corrections, respectively, when using a slightly conservative weight of $20 \mathrm{~mm}$ per normal point. Position and bias corrections themselves are well above the 1-cm level for the majority of stations and even reach the decimeter range for a few sites. When applying the respective corrections in the residuals computation a remarkable factor-of-two improvement in the RMS residuals can be noted (Table 5) that clearly evidences the significance of the derived corrections and underlines obvious deficiencies in the employed SLRF2008 site coordinates and/or range calibrations.

Table 5 SLR residuals (mean \pm standard deviation) of Swarm-C, TerraSAR-X, Sentinel-3A, and Jason-2 ambiguity-fixed orbits before and after the adjustment of station coordinates and range biases. A common threshold of $0.2 \mathrm{~m}$ and an elevation mask of $10^{\circ}$ were applied in all cases.

\begin{tabular}{lccc}
\hline Coordinates & $\begin{array}{c}\text { Uncorrected } \\
{[\mathrm{mm}]}\end{array}$ & $\begin{array}{c}\text { Corrected } \\
{[\mathrm{mm}]}\end{array}$ & Stations \\
\hline SLRF2008 & $-1.4 \pm 24.2$ & $0.0 \pm 11.7$ & 32 \\
SLRF2014 & $-1.9 \pm 16.5$ & $0.0 \pm 11.7$ & 32 \\
\hline
\end{tabular}

For comparison, we have therefore repeated the analysis using SLRF2014 site coordinates, which turn out to provide a notably better consistency with observations (Table 5). Even though the SLRF2014 is formally "incompatible" with the IGb08 reference frame of the GPS-based LEO orbit products, it evidently provides much better coordinates for the period of interest (i.e., 2016). While this is easily understood for a station like Arequipa that lacks post-earthquake information in SLRF2008 and thus requires an $11 \mathrm{~cm}$ coordinate correction, most other stations also benefit from the use of SLRF2014 coordinates as well. While the median of the position corrections for all stations amounts to $30 \mathrm{~mm}$ for SLR2008, it reduces to $13 \mathrm{~mm}$ when working with SLRF2014 a priori coordinates. These findings are in line with the results of Zelensky et al (2018) who showed by how much ITRF2008 deteriorates when extrapolated past the 2009 solution end.

An overview of position and range bias corrections for SLRF2014 is provided in Table 6. Aside from the removal of a mean bias, clear improvements in the standard deviation may be recognized for at least five stations (Arkhyz, Brazilia, Irkutsk, Katzively, and Riga) when using the revised site coordinates and range bias corrections. For three 
Table 6 Estimated SLRF2014 position corrections in east (E), north (N), and up (U) direction and range biases (b) with formal uncertainties. In addition, the number of normal point $\left(n_{\mathrm{np}}\right)$ and the residuals before/after applying the respective corrections are given. All values are based on data for Jan.-Dec. 2016 and thus refer to the mean epoch 2016.5. SLR residuals of Swarm-C, TerraSAR-X, Sentinel-3A, and Jason-2 were used. No timing bias corrections were applied.

\begin{tabular}{|c|c|c|c|c|c|c|c|}
\hline Station & SOD & $\mathrm{E}[\mathrm{mm}]$ & $\mathrm{N}[\mathrm{mm}]$ & $\mathrm{U}[\mathrm{mm}]$ & $b[\mathrm{~mm}]$ & $n_{\mathrm{np}}$ & Residuals [mm] \\
\hline Arequipa & 74031306 & $2.7 \pm 0.6$ & $4.0 \pm 0.6$ & $12.9 \pm 2.0$ & $12.7 \pm 1.2$ & 3674 & $5.8 \pm 11.9 /-0.0 \pm 11.4$ \\
\hline Arkhyz & 18869601 & $6.9 \pm 1.6$ & $-4.9 \pm 1.8$ & $-143.8 \pm 4.8$ & $-87.9 \pm 2.9$ & 614 & $-16.4 \pm 28.5 /-0.0 \pm 14.3$ \\
\hline Badary & 18900901 & $-3.7 \pm 0.8$ & $-3.4 \pm 0.7$ & $6.6 \pm 2.6$ & $5.4 \pm 1.7$ & 2455 & $1.7 \pm 17.6 /-0.0 \pm 17.3$ \\
\hline Beijing & 72496102 & $3.8 \pm 1.1$ & $6.0 \pm 1.2$ & $20.7 \pm 3.3$ & $5.1 \pm 2.1$ & 1105 & $-7.9 \pm 15.6 / \quad 0.0 \pm 14.8$ \\
\hline Borowiec & 78113802 & $1.5 \pm 1.2$ & $-0.3 \pm 1.1$ & $64.8 \pm 3.4$ & $1.6 \pm 2.2$ & 1112 & $-37.4 \pm 26.8 /-0.0 \pm 24.2$ \\
\hline Brazilia & 74072701 & $-32.6 \pm 2.6$ & $-10.4 \pm 2.1$ & $8.2 \pm 8.5$ & $8.5 \pm 4.6$ & 228 & $-1.8 \pm 21.8 / 0.0 \pm 13.1$ \\
\hline Changchun & 72371901 & $-2.0 \pm 0.3$ & $1.4 \pm 0.3$ & $26.4 \pm 0.8$ & $4.6 \pm 0.4$ & 16961 & $-8.8 \pm 21.9 / \quad 0.0 \pm 21.2$ \\
\hline Grasse & 78457801 & $-2.7 \pm 0.3$ & $-3.6 \pm 0.2$ & $-0.7 \pm 0.7$ & $-18.2 \pm 0.4$ & 19300 & $-16.9 \pm 11.5 / \quad 0.0 \pm 11.2$ \\
\hline Graz & 78393402 & $1.9 \pm 0.3$ & $-1.7 \pm 0.3$ & $3.3 \pm 0.9$ & $1.7 \pm 0.5$ & 13168 & $0.4 \pm 7.3 /-0.0 \pm 7.1$ \\
\hline Greenbelt & 71050725 & $8.4 \pm 0.2$ & $4.4 \pm 0.3$ & $-8.5 \pm 0.8$ & $-11.3 \pm 0.4$ & 17438 & $-7.5 \pm 10.5 /-0.0 \pm 8.5$ \\
\hline Haleakala & 71191402 & $4.5 \pm 0.5$ & $5.4 \pm 0.5$ & $-2.9 \pm 1.7$ & $8.1 \pm 1.0$ & 5277 & $10.5 \pm 9.1 / \quad 0.0 \pm 8.4$ \\
\hline Hartebeesthoek & 75010602 & $-2.4 \pm 0.6$ & $-0.5 \pm 0.6$ & $-1.8 \pm 2.1$ & $-6.9 \pm 1.3$ & 3322 & $-5.9 \pm 12.4 /-0.0 \pm 12.3$ \\
\hline Herstmonceux & 78403501 & $1.2 \pm 0.3$ & $-2.0 \pm 0.3$ & $-3.2 \pm 1.2$ & $-3.2 \pm 0.8$ & 13391 & $-0.8 \pm 6.8 /-0.0 \pm 6.7$ \\
\hline Irkutsk & 18915301 & $15.7 \pm 1.4$ & $14.5 \pm 1.3$ & $20.3 \pm 4.7$ & $9.4 \pm 3.2$ & 835 & $-7.3 \pm 17.5 /-0.0 \pm 13.4$ \\
\hline Katzively & 18931801 & $0.7 \pm 0.7$ & $-16.7 \pm 0.8$ & $-71.8 \pm 2.6$ & $-68.2 \pm 1.6$ & 2483 & $-23.9 \pm 28.6 /-0.0 \pm 25.6$ \\
\hline Matera & 79417701 & $0.6 \pm 0.6$ & $-3.2 \pm 0.6$ & $-3.9 \pm 2.3$ & $-9.0 \pm 1.3$ & 3521 & $-6.3 \pm 8.2 /-0.0 \pm 7.9$ \\
\hline McDonald & 70802419 & $10.2 \pm 2.4$ & $5.3 \pm 3.1$ & $16.9 \pm 8.5$ & $-15.3 \pm 5.7$ & 214 & $-26.9 \pm 19.5 /-0.0 \pm 18.4$ \\
\hline Mendeleevo 2 & 18748301 & $1.4 \pm 1.4$ & $-3.9 \pm 1.5$ & $2.5 \pm 5.2$ & $-7.3 \pm 3.6$ & 882 & $-7.6 \pm 13.5 / \quad 0.0 \pm 13.4$ \\
\hline Monument Peak & 71100412 & $7.6 \pm 0.3$ & $-7.1 \pm 0.3$ & $-12.2 \pm 1.0$ & $-2.2 \pm 0.6$ & 14329 & $5.4 \pm 12.5 /-0.0 \pm 11.0$ \\
\hline Mount Stromlo & 78259001 & $1.7 \pm 0.3$ & $-0.2 \pm 0.3$ & $0.5 \pm 0.9$ & $3.2 \pm 0.6$ & 17274 & $3.0 \pm 11.7 /-0.0 \pm 11.7$ \\
\hline Papeete & 71240802 & $12.2 \pm 0.8$ & $-7.8 \pm 0.7$ & $-26.8 \pm 2.3$ & $-17.4 \pm 1.4$ & 2572 & $-0.6 \pm 29.9 /-0.0 \pm 28.5$ \\
\hline Potsdam & 78418701 & $-0.6 \pm 0.4$ & $-2.9 \pm 0.3$ & $15.6 \pm 1.1$ & $1.4 \pm 0.7$ & 11477 & $-7.7 \pm 10.1 / \quad-0.0 \pm 9.6$ \\
\hline Riga & 18844401 & $-8.8 \pm 0.9$ & $-11.6 \pm 0.8$ & $185.2 \pm 2.3$ & $172.9 \pm 1.5$ & 2191 & $60.2 \pm 39.5 /-0.0 \pm 14.0$ \\
\hline Sejong & 73942601 & $4.4 \pm 1.3$ & $4.7 \pm 1.3$ & $-3.1 \pm 4.3$ & $6.1 \pm 3.0$ & 949 & $7.7 \pm 19.6 / 0.0 \pm 19.3$ \\
\hline Shanghai & 78212801 & $0.7 \pm 0.7$ & $1.9 \pm 0.7$ & $8.0 \pm 2.1$ & $-11.3 \pm 1.2$ & 2734 & $-15.8 \pm 13.1 /-0.0 \pm 13.0$ \\
\hline Simosato & 78383603 & $3.9 \pm 0.8$ & $-4.6 \pm 0.8$ & $-31.4 \pm 2.6$ & $-10.6 \pm 1.6$ & 2214 & $8.8 \pm 11.9 / \quad 0.0 \pm 10.3$ \\
\hline Svetloe & 18889801 & $1.7 \pm 0.8$ & $3.0 \pm 1.0$ & $-8.1 \pm 2.6$ & $-5.1 \pm 1.8$ & 2072 & $0.1 \pm 17.6 / \quad 0.0 \pm 17.5$ \\
\hline Wettzell (SOSW) & 78272201 & $-1.1 \pm 0.8$ & $-10.8 \pm 0.7$ & $-8.4 \pm 2.4$ & $2.4 \pm 1.5$ & 2629 & $9.8 \pm 10.2 / \quad 0.0 \pm 8.3$ \\
\hline Wettzell (WLRS) & 88341001 & $1.0 \pm 0.6$ & $-2.0 \pm 0.6$ & $-5.8 \pm 1.9$ & $-25.5 \pm 1.3$ & 4376 & $-21.2 \pm 9.1 /$ \\
\hline Yarragadee & 70900513 & $1.2 \pm 0.1$ & $-2.2 \pm 0.1$ & $-3.3 \pm 0.5$ & $2.2 \pm 0.2$ & 55586 & $3.4 \pm 8.1 / \quad 0.0 \pm 7.9$ \\
\hline Zelenchukskya & 18899901 & $13.6 \pm 1.1$ & $-3.1 \pm 1.3$ & $-22.8 \pm 4.5$ & $-6.8 \pm 2.9$ & 990 & $8.9 \pm 19.4 /-0.0 \pm 17.8$ \\
\hline Zimmerwald & 78106801 & $1.5 \pm 0.4$ & $-1.6 \pm 0.4$ & $8.4 \pm 1.3$ & $1.0 \pm 0.7$ & 7814 & $-3.0 \pm 6.7 /-0.0 \pm 6.4$ \\
\hline
\end{tabular}

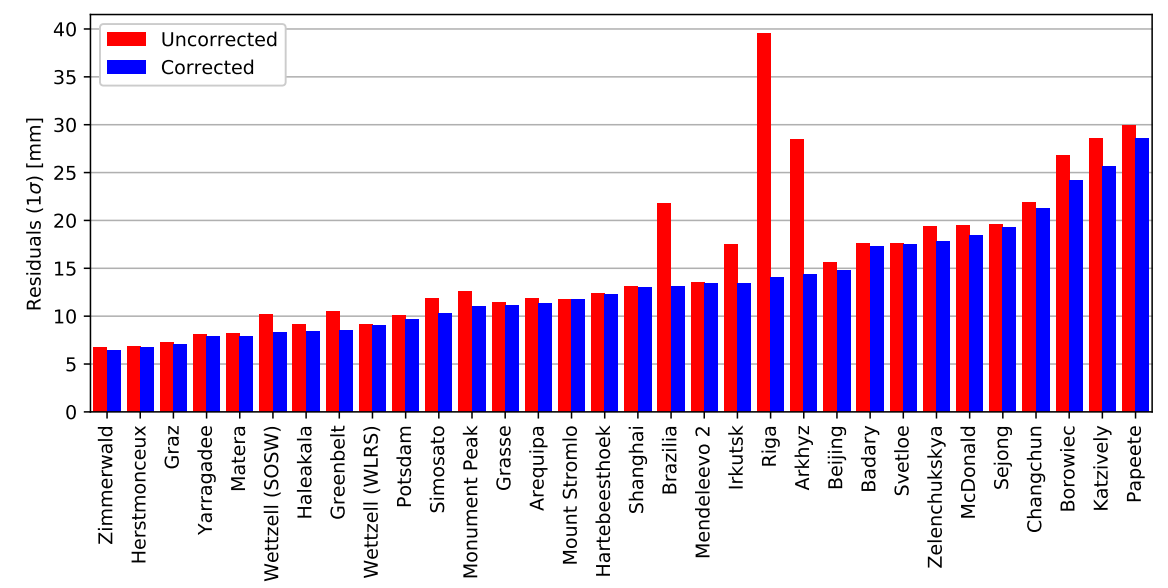

Fig. 5 SLR residuals of Swarm-C, TerraSAR-X, Sentinel-3A, and Jason-2 ambiguity-fixed orbits for individual ILRS stations before and after correction of SLRF2014 site coordinates and station-specific range biases. 


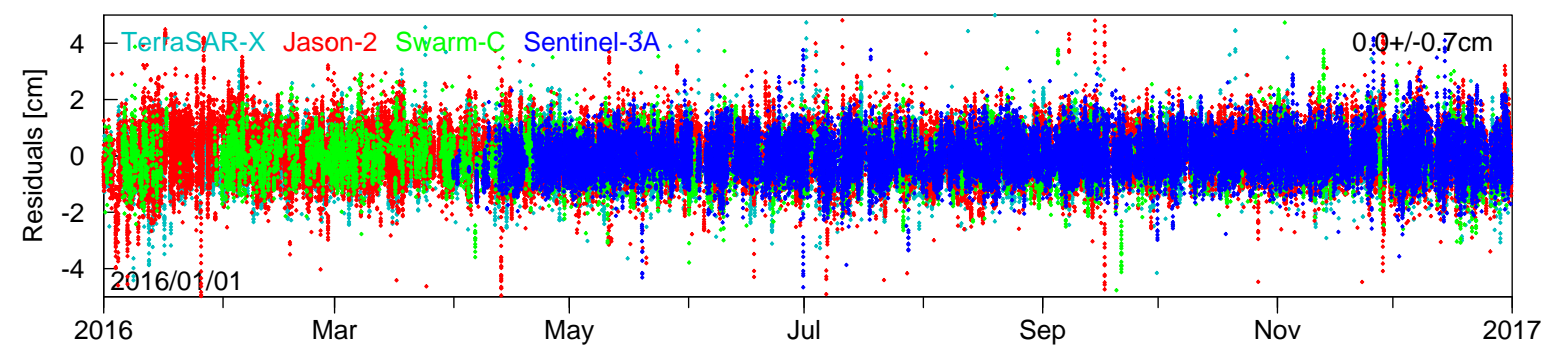

Fig. 6 SLR residuals of Swarm-C, TerraSAR-X, Sentinel-3A, and Jason-2 ambiguity-fixed orbits for 12 high-performance stations after correction of site coordinates and station-specific range biases. An outlier threshold of $5 \mathrm{~cm}$ was applied.
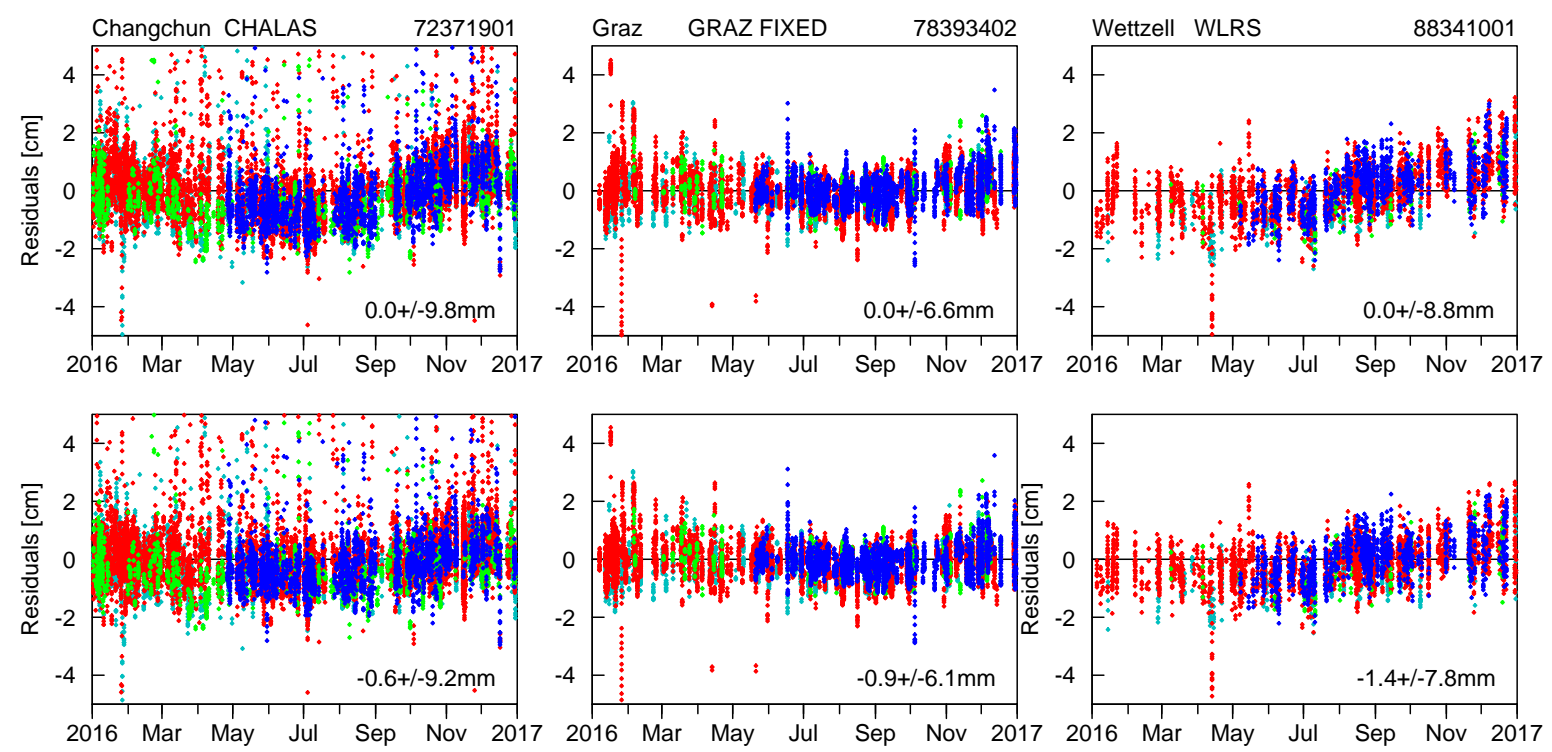

Fig. 7 SLR residuals of Swarm-C (green), TerraSAR-X (black), Sentinel-3A (blue), and Jason-2 (red) orbits for three selected ILRS stations without (top) and with (bottom) consideration of atmospheric pressure loading. Station coordinates and range biases are corrected with values from Table 6 and outliers exceeding a $5 \mathrm{~cm}$ threshold were discarded. Values in the lower right corner of each subfigure indicate the mean value and standard deviation of the respective SLR residuals

of these (Arkhyz, Katzively, and Riga) strikingly large height corrections and associated range biases of $7-20 \mathrm{~cm}$ are inferred from the elevation dependence of the SLR residuals. These large height corrections are consistent to height corrections obtained independently in an ILRS-like processing of SLR observations to the laser geodetic satellites LAGEOS1 and -2 , provided that range bias parameters are estimated for all stations (K. Sośnica, priv. comm.). While the exact cause of these discrepancies cannot be identified without further information of the station operator, an improper choice of the reference point for ranging measurements (typically the intersection of the two axes of the telescope mount) is considered as a potential explanation.

A reduction of SLR residuals by more than $10 \%$ in terms of standard deviation can also be recognized for a couple of high-performance stations such as Greenbelt, Monument Peak, and Wettzell (SOSW). Even though the position (and bias) corrections for these stations are much smaller than for the sites discussed before, the corrections still appear statistically significant. A review of the ranging system calibration and relevant local ties is encouraged to best exploit the superior ranging quality of the stations.

As illustrated in Fig. 5, the majority of stations offers a ranging precision of better than $20 \mathrm{~mm}$, and a substantial fraction of stations achieves a standard deviation in the $5-10 \mathrm{~mm}$ range. Aside from the ranging noise, which is particularly low for stations using kHz laser systems (e.g., Graz, Herstmonceux; Gibbs et al 2006), these values include a variety of possible measurement and modeling errors related to, e.g., time varying range calibration errors, timing, atmospheric tides, or tropospheric delays, and, finally, the errors of the employed LEO satellite orbits. On average over the previously selected set of 12 high performance stations, SLR residuals of $7 \mathrm{~mm}$ standard deviation are obtained with the 
present orbit products (Fig. 6). For individual missions the RMS residuals range from $6.5 \mathrm{~mm}$ (Sentinel-3A) to $7.5 \mathrm{~mm}$ (Swarm-C).

Making use of other (non-ambiguity-fixed) orbit products from Table 2, similar station coordinate corrections and range biases can be obtained. However, the results suffer from potential inconsistencies in the underlying reference frames due to the use of different GPS orbit products and/or the mix of different radiometric techniques (i.e., joint use of GPS and DORIS in the Jason-2 GDR-E products). Furthermore, the benefit of station coordinate and range bias adjustments for the SLR modeling is less obvious in that case due to the higher overall residuals level.

\subsubsection{Loading effects}

For illustration of long-term variations in the ranging performance, residuals for a subset of ILRS stations are presented in Fig. 7. Over the one-year analysis period, variations in the mean residuals over time scales of several months and peakto-peak amplitudes of about 1-2 $\mathrm{cm}$ may be recognized for the selected sites, which indicate seasonal variation in the range biases and/or the station coordinate modeling.

Possible explanations include Earth crust deformations due to land hydrology and atmospheric loading, both of which have been neglected in the standard modeling of station coordinates discussed so far. To assess the impact of these effects, we independently evaluated the effects of atmospheric pressure loading (APL) on the SLR residuals based on the Vienna APL products (Wijaya et al 2013; GGOSATM 2017) for 2016. Among the deformation models neglected in our standard processing, APL is considered the leading contribution and may cause $\mathrm{cm}$-level peak variations in the station coordinates (Dach et al 2011). Average annual signals are notably smaller, though, and range from $1-5 \mathrm{~mm}$ for stations of the ILRS network (Sośnica et al 2013; Bury and Sośnica 2017).

This is generally below the overall precision of the SLR measurements and their modeling, and only subtle effects can be recognized in the SLR residuals statistics. Along with a reduction of the standard deviation by about $0.5-1 \mathrm{~mm}$, changes of similar magnitude are also obtained in the mean values. The latter is related to weather restrictions in the distribution of SLR observations and known as the "blue-sky" effect (Otsubo et al 2004). On the other hand, consideration of APL does not or only partly remove the dominant seasonal trends observed in the residuals of many stations. Within the present study, discernible benefits were only observed for the Changchun station were the annual signal in the residuals is visibly flattened (see Fig. 7, left).

Obviously, APL alone is unable to explain the observed seasonal variations in the SLR residuals. Since consistent time series are obtained for all four LEO satellites, either station-specific range bias calibration uncertainties or small systematic errors in the GPS orbit products (Loyer et al 2012) used for our LEO POD are considered as plausible explanation for the remaining seasonal errors in the SLR observation modeling.

\subsubsection{Timing biases}

Aside from range biases and station position errors, the SLR measurements may also be affected by timing errors at the stations. Systematic biases in the station time do not affect measurements of the turnaround time of laser pulses, but result in erroneous measurement time tags. These will in turn cause range modeling errors $\Delta \rho_{t}$ proportional to the line-ofsight velocity and the timing offset itself.

By way of example, a $1 \mu \mathrm{s}$ bias in the measurement epoch will show up as a variation of up to $\pm 8 \mathrm{~mm}$ in the range residuals across a single pass of a LEO satellite. Given the availability of GPS disciplined clocks, sub- $\mu$ s timing accuracy should be expected for a properly maintained laser ranging station. Nevertheless, timing errors of up to a few $\mu \mathrm{s}$ as well as notable jumps or drifts were revealed within the ILRS network by Exertier et al (2017) based on data of the Jason-2 "Time Transfer by Laser Link" (T2L2) experiment between 2013 and 2016.
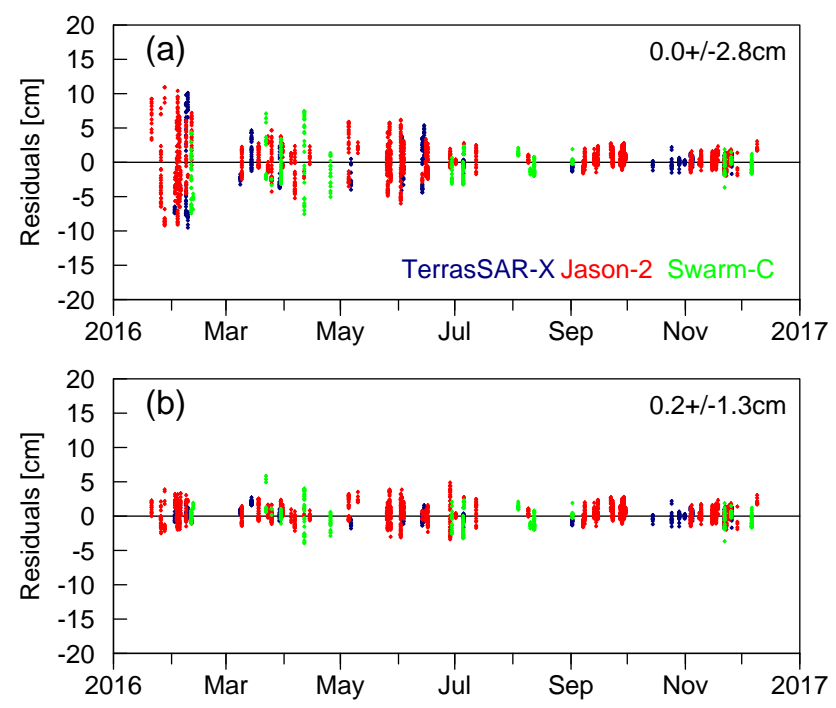

Fig. 8 SLR residuals of the Papeete (MOBLAS-8) station before (a) and after (b) application of timing biases of $-14.4 \mu$ s for January to February and $-5.8 \mu$ s from March to June 2016

As outlined in Sect. 3.3, timing offsets can also be estimated from SLR residuals of LEO satellites. To reduce the impact of possible along-track satellite position offsets, we jointly processed data from the four aforementioned missions over the entire year 2016. Within this analysis oneyear-average timing biases at the sub- $\mu$ s level were obtained 
for most ILRS stations with formal uncertainties of 0.1$0.2 \mu \mathrm{s}$. In general, application of the estimated biases shows at best a marginal (approx. $0.1 \mathrm{~mm}$ ) reduction of the RMS residuals of individual stations. While shorter analysis intervals might help to better take into account the time variability of the timing biases, this would also further reduce their statistical significance.

As an exception to the above significant biases were obtained for the Papeete station, that show up as exceptionally large residuals in the first half of the year. Here, timing biases of $-14.4 \mu \mathrm{s}$ for January to February and $-5.8 \mu \mathrm{s}$ for March to June could be derived that yield a notably improved ranging performance when taken into account in the observation modeling (Fig. 8). Even though a direct comparison with Exertier et al (2017) is hampered by different analysis periods, time biases of similar magnitude have also been reported for Papeete in that work.

Independent timing biases are routinely derived on a perpass basis by Otsubo (2017) from post fit residuals of low and high altitude satellites as part of a low-latency quality control process. Despite a larger scatter of timing biases obtained from individual laser ranging satellites, the results are, on average, in good accordance with our results for the Papeete station (Table 7).

Table 7 Average time biases of Papeete from quick-look quality reports of Otsubo (2017) for selected laser ranging satellites in 2016. All values in $\mu \mathrm{s}$

\begin{tabular}{lcc}
\hline Analysis & Jan-Feb & Mar-Apr \\
\hline Laser ranging satellites (Otsubo 2017) & & \\
LAGEOS-1 & -13.0 & -6.3 \\
LAGEOS-2 & -8.3 & -8.1 \\
LARES & -12.2 & -4.1 \\
Starlette & -22.1 & -6.3 \\
Mean & -14.1 & -6.1 \\
LEOs (this study) & -14.4 & -6.0 \\
\hline
\end{tabular}

Aside from this particular case, the effect of timing errors is largely buried under other error contributions in our analysis of the ILRS network and therefore most likely negligible in most current applications. Careful attention to timing offset calibrations should nevertheless be paid by station operators to ensure the highest quality of SLR observations.

\subsection{SLR Tracking of Satellite Formations}

The LEO missions currently supported by ILRS tracking include three formation flying missions, where two satellites orbit the Earth at small separations compared to their orbital radius (Sect. 2). Distances between the spacecraft range from less than a kilometer for TanDEM-X to $100-200 \mathrm{~km}$ for Swarm and GRACE. The two satellites in each of the formations thus share an almost common visibility over the SLR stations with maximum time offsets of less than $30 \mathrm{~s}$. Interleaved tracking is supported by a variety of ILRS stations that can quickly switch between targets during a single pass.

Given the availability of GPS receivers with a common field of view, relative positions (or "baselines") can be computed for all of the aforementioned missions using differential carrier phase observations (Kroes et al 2005; Jäggi et al 2012; Allende-Alba et al 2017). Other than absolute positions of single-satellite POD which commonly achieve a cm-level accuracy, the carrier phase based relative positions were independently verified to exhibit mm-level accuracy for at least two of these missions using K-band ranging (GRACE) or interferometric SAR analysis (TanDEM$\mathrm{X})$. As such, SLR tracking of formation flying missions can shed further light on the performance of current ILRS stations.

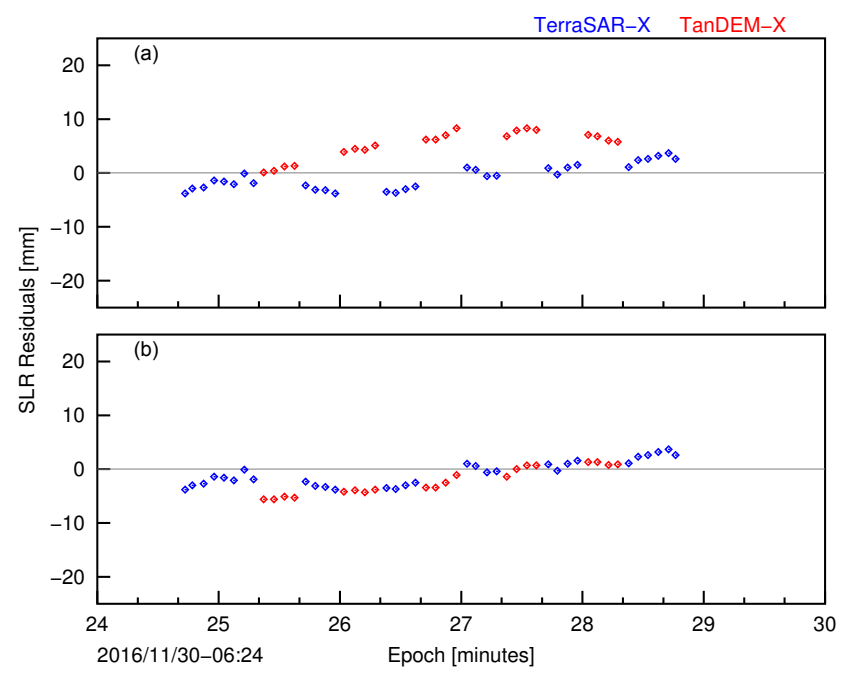

Fig. 9 SLR residuals of the TerraSAR-X and TanDEM-X satellites over a single pass of interleaved tracking from the Herstmonceux station: (a) based on independent precise orbit determination solutions of the two satellites and (b) based on combination of a TerraSAR-X POD with a carrier phase differential navigation solution for the TerraSAR$\mathrm{X} / \mathrm{TanDEM}-\mathrm{X}$ baseline

To illustrate this specialized application of LEO SLR tracking, a sample set of SLR residuals for the TerraSAR$\mathrm{X} /$ TanDEM-X formation is shown in Fig. 9. The figure covers a single pass of tracking from the Herstmonceux station collected in late November 2016. Tracking started with TerraSAR-X, and alternated between both spacecraft every $20 \mathrm{~s}$. During each of these intervals a total of four $5 \mathrm{~s}$ normal points were collected. The use of a $\mathrm{kHz}$ laser system enables a large number of 200-500 individual measurements per normal point and thus a (sub)-mm precision of each normal point. 
This high precision is readily evidenced by a very small scatter of consecutive normal points for the two tracked satellites. On the other hand, differences of about $1 \mathrm{~cm}$ may be recognized (Fig. 9a) for the residuals computed from independent, non-ambiguity resolved POD solutions for the two satellites. This is consistent with independent assessments of the relative navigation performance of differenced singlesatellite orbit determination results reported, e.g., in Jäggi et al (2007b) and Montenbruck et al (2017a).

When using ambiguity-fixed, differential carrier phase observations for the baseline determination of the TerraSAR$\mathrm{X}$ / TanDEM-X formation, a notably increased consistency of SLR residuals for the two spacecraft is obtained (Fig. 9b). It confirms both a high precision of the baseline determination process, but also an excellent precision of the laser tracking system, when toggling between close-by targets. Even though a detailed quantitative analysis is beyond the scope of the present study, the example nicely illustrates an interesting opportunity for validating advanced SLR tracking systems and/or the baseline determination performance of formation flying missions in low Earth orbit.

\section{Summary and Conclusions}

As a fully independent metrology system, satellite laser ranging (SLR) to low Earth orbiters (LEOs) represents an indispensable tool for validating GPS-based precise orbit determination (POD) solutions. The validation is based on SLR residuals that represent the differences between observed and modeled station-to-satellite ranges. These demonstrate a consistency of present LEO orbit products with the optical measurements at a level of $1-3 \mathrm{~cm}$ and help to demonstrate conformance of these products with mission requirements.

Based on the SLR observations, the relative performance of different processing schemes for GPS-based POD may be assessed. Even though the continuous coverage and the availability of observations from multiple GPS satellites at each epoch enable a purely kinematic positioning of LEO satellites, dynamic or reduced-dynamic orbit models generally result in smoother orbits and improved consistency with SLR observations. Compared to the estimation of floatvalued ambiguities, the use of single-receiver ambiguity fixing is likewise found to benefit the orbit determination process.

Beyond a straightforward use of SLR residuals statistics as a quality metrics for LEO POD solutions, satellite laser ranging is useful to identify systematic orbit errors that may result from deficiencies of the dynamical orbit model or improper information on the accommodation of sensors and antennas. Vice versa, high-quality LEO orbits may serve as reference for improving station positions and bias calibrations in the laser tracking network. Besides the use of local ties at suitably equipped fundamental sites, the SLR validation of GPS-based LEO orbits thus offers a complementary approach to integrate different space geodetic techniques in the construction of global reference frames.

The present study demonstrates a clear benefit of newly available SLRF2014 site coordinates over SLRF2008 values for the analysis of LEO orbits in the year 2016, even though these orbits still refer to the ITRF2008-compatible reference frame realization (IGb08) of the International GNSS Service (IGS). Nevertheless, notable corrections of site coordinates and range biases can be identified for various sites that result in a clearly improved modeling of the SLR observations.

It must be stated, though, that systematic orbit and station errors can affect the modeled SLR range in a similar manner and may thus be difficult to disentangle in the analysis of SLR residuals. Radial orbit errors, station height errors, and range biases, in particular, result in highly correlated contributions that cannot be separated without a proper calibration of the laser tracking network, or accurate knowledge of the relative locations of the laser retroreflector array, GPS antenna phase center and center-of-mass onboard the LEO satellite. Due attention also needs to be paid to the modeling of line-of-sight dependent range variations related to the use of multi-prism retro-reflector arrays. In this context, the use of fully two-dimensional range corrections is strongly suggested for all LRAs and LEO missions.

Overall, a 5-10 $\mathrm{mm}$ precision (repeatability) of SLR residuals can presently be achieved for high-grade stations of the International Laser Ranging Service network when making use of state-of-the-art LEO orbit products. Observed inconsistencies in the onboard coordinates of LRA, phase center and CoM are of similar magnitude and the same applies to ranging biases and coordinate uncertainties of many ILRS stations. Continued effort for proper calibration of the ground network and onboard systems will thus be required to remove systematic errors and to turn the precision of today's laser ranging system into a similar level of accuracy. To fully exploit the ranging quality of the SLR stations, operators should pay particular attention to a proper definition of the relevant reference points and local ties, as well as to timing offset calibrations.

Acknowledgements The authors are grateful to all members of the International Satellite Laser Ranging Service for their continued effort to collect and publicly provide SLR observations of geodetic satellites. Their work provides the basis for the present study and is an essential contribution to numerous space missions. We also acknowledge the use of various precise orbit determination products of low Earth orbit satellites shared by the Centre National d'Etudes Spatiales, the Copernicus POD Service, the European Space Agency ESA, the University of Texas, the Jet Propulsion Laboratory, and various other institutions. Their support is likewise highly appreciated. 


\section{References}

Allende-Alba G, Montenbruck O, Jäggi A, Arnold D, Zangerl F (2017) Reduced-dynamic and kinematic baseline determination for the Swarm mission. GPS Solutions pp 1-10, DOI 10.1007/ s10291-017-0611-z

Altamimi Z, Rebischung P, Métivier L, Collilieux X (2016) ITRF2014: A new release of the International Terrestrial Reference Frame modeling nonlinear station motions. Journal of Geophysical Research: Solid Earth 121(8):6109-6131, DOI 10.1002/ 2016JB013098

Ashby N (2004) The Sagnac effect in the Global Positioning System. In: Relativity in Rotating Frames, Springer, pp 11-28, DOI 10. 1007/978-94-017-0528-8_3

Auriol A, Tourain C (2010) DORIS system: the new age. Advances in Space Research 46(12):1484-1496, DOI 10.1016/j.asr.2010.05. 015

Bertiger W, Desai SD, Haines B, Harvey N, Moore AW, Owen S, Weiss JP (2010) Single receiver phase ambiguity resolution with GPS data. Journal of Geodesy 84(5):327-337, DOI 10.1007/ s00190-010-0371-9

Bettadpur S (2012) Gravity recovery and climate experiment - product specification document, 4.6 (edn). URL ftp: //podaac.jpl.nasa.gov/allData/grace/ docs/ProdSpecDoc_v4.6.pdf

Bock H, Jäggi A, Beutler G, Meyer U (2014) GOCE: precise orbit determination for the entire mission. Journal of Geodesy 88(11):1047-1060, DOI 10.1007/s00190-014-0742-8

Boomkamp H (2003) The CHAMP orbit comparison campaign. In: First CHAMP Mission Results for Gravity, Magnetic and Atmospheric Studies, Springer, pp 53-58

Buckreuss S, Balzer W, Mühlbauer P, Werninghaus R, Pitz W (2003) The TerraSAR-X satellite project. In: Proc. Int. Geoscience and Remote Sensing Symposium IGARSS'03, IEEE, vol 5, pp 30963098, DOI 10.1109/IGARSS.2003.1294694

Bury G, Sośnica K (2017) Impact of atmospheric pressure loading on SLR-derived station coordinates using range measurements to multi-GNSS satellites. In: Proc. ILRS Technical Workshop 2017, Riga, Latvia, October 02-05, 2017

Chalmers OSO (2016) Ocean tide loading provider web site. URL http://holt.oso. chalmers.se/loading/

CNES (2015) SP3 orbits JASON-2. URL ftp://cddis.gsfc. nasa.gov/doris/products/orbits/ssa/ja2/ README_SP 3_ja2.txt

Combrinck L (2010) Satellite laser ranging. In: Sciences of Geodesy, vol 1, Springer, pp 301-338, DOI 10.1007/978-3-642-11741-1_9

Coulot D, Berio P, Biancale R, Loyer S, Soudarin L, Gontier AM (2007) Toward a direct combination of space-geodetic techniques at the measurement level: Methodology and main issues. Journal of Geophysical Research: Solid Earth 112(B5), DOI 10.1029/ 2006JB004336

Dach R, Böhm J, Lutz S, Steigenberger P, Beutler G (2011) Evaluation of the impact of atmospheric pressure loading modeling on GNSS data analysis. Journal of geodesy 85(2):75-91

Donlon C, Berruti B, Buongiorno A, Ferreira MH, Féménias P, Frerick J, Goryl P, Klein U, Laur H, Mavrocordatos C, et al (2012) The global monitoring for environment and security (GMES) Sentinel3 mission. Remote Sensing of Environment 120:37-57, DOI 10. 1016/j.rse.2011.07.024

Dow JM, Neilan RE, Rizos C (2009) The international GNSS service in a changing landscape of global navigation satellite systems. Journal of Geodesy 83(3):191-198, DOI 10.1007/ s00190-008-0300-3

Dunn C, Bertiger W, Franklin G, Harris I, Kruizinga G, Meehan T, Nandi S, Nguyen D, Rogstad T, Thomas JB, Tien J (2002) The instrument on NASA's GRACE mission: augmentation of GPS to achieve unprecedented gravity field measurements. Proceedings of the 15th International Technical Meeting of the Satellite Division of The Institute of Navigation (ION GPS 2002) pp 724-730

Exertier P, Belli A, Lemoine JM (2017) Time biases in laser ranging observations: A concerning issue of space geodesy. Advances in Space Research 60:948-968, DOI 10.1016/j.asr.2017.05.016

Fernández J, Fernández C, Féménias P, Peter H (2016) The Copernicus Sentinel-3 mission. In: ILRS Workshop 2016, ILRS, pp 1-4

Fletcher K (ed) (2012) Sentinel-3 - ESA's Global Land and Ocean Mission for GMES Operational Services. ESA SP-1322/3, Noordwijk

Floberghagen R, Fehringer M, Lamarre D, Muzi D, Frommknecht B, Steiger C, Piñeiro J, Da Costa A (2011) Mission design, operation and exploitation of the gravity field and steady-state ocean circulation explorer mission. Journal of Geodesy 85(11):749-758, DOI 10.1007/s00190-011-0498-3

Flohrer C, Otten M, Springer T, Dow J (2011) Generating precise and homogeneous orbits for Jason-1 and Jason-2. Advances in Space Research 48(1):152-172, DOI 10.1016/j.asr.2011.02.017

Friis-Christensen E, Lühr H, Knudsen D, Haagmans R (2008) Swarm - an earth observation mission investigating geospace. Advances in Space Research 41(1):210-216, DOI 10.1016/j.asr.2006.10.008

GGOSATM (2017) Vienna-APL data sets. URL http : / / ggosatm . hg.tuwien.ac . at/LOADING/VERSION4/GRID/

Gibbs P, Potter C, Sherwood R, Wilkinson M, Benham D, Smith V, Appleby G (2006) Some early results of kilohertz laser ranging at Herstmonceux. In: Proc. 15th international workshop on laser ranging, pp 250-258

Hackel S, Montenbruck O, Steigenberger P, Balss U, Gisinger C, Eineder M (2017) Model improvements and validation of TerraSAR-X precise orbit determination. Journal of Geodesy 91(5):547-562, DOI 10.1007/s00190-016-0982-X

Haines BJ, Armatys MJ, Bar-Sever YE, Bertiger WI, Desai SD, Dorsey AR, Lane CM, Weiss JP (2011) One-centimeter orbits in near-real time: the GPS experience on OSTM/Jason-2. The Journal of the Astronautical Sciences 58(3):445-459, DOI 10.1007/ BF03321179

Holt G, Lightsey E, Montenbruck O (2003) Benchmark testing for spaceborne global positioning system receivers. In: AIAA Guidance, Navigation, and Control Conference and Exhibit, p 5666

IDS (2015) New GDR-E orbit standards. URL ftp: / / ftp.ids-doris.org/pub/ids/data/POD_ configuration_GDRE.pdf

van den IJssel J, Encarnação J, Doornbos E, Visser P (2015) Precise science orbits for the Swarm satellite constellation. Advances in Space Research 56(6):1042-1055, DOI 10.1016/j.asr.2015.06.002

ILRS (2017) SLRF2014 station coordinates. URL ftp://ftp. cddis.eosdis.nasa.gov/pub/slr/products/ resource/SLRF2014_POS+VEL_2030.0_170605.snx

ILRS Analysis Standing Committee (2016) SLRF2008. URL https://ilrs.cddis.eosdis.nasa.gov/science/ awg/SLRF2008.html

ITRF (2017) ITRF2014 post-seismic deformation data for SLR stations. URL ftp://itrf.ign.fr/pub/itrf/itrf2014/ ITRF 2014-psd-slr.dat

Jäggi A, Hugentobler U, Beutler G (2006) Pseudo-stochastic orbit modeling techniques for low-Earth orbiters. Journal of Geodesy 80(1):47-60, DOI 10.1007/s00190--006--0029--9

Jäggi A, Beutler G, Bock H, Hugentobler U (2007a) Kinematic and highly reduced-dynamic LEO orbit determination for gravity field estimation. In: Rizos C, Tregoning P (eds) Dynamic Planet Monitoring and Understanding a Dynamic Planet with Geodetic and Oceanographic Tools, Springer, pp 354-361, DOI 10.1007/ 978-3-540-49350-1_52

Jäggi A, Hugentobler U, Bock H, Beutler G (2007b) Precise orbit determination for GRACE using undifferenced or doubly differenced GPS data. Advances in Space Research 39(10):1612-1619, DOI 
10.1016/j.asr.2007.03.012

Jäggi A, Dach R, Montenbruck O, Hugentobler U, Bock H, Beutler G (2009) Phase center modeling for LEO GPS receiver antennas and its impact on precise orbit determination. Journal of Geodesy 83(12):1145-1162, DOI 10.1007/s00190-009-0333-2

Jäggi A, Montenbruck O, Moon Y, Wermuth M, König R, Michalak G, Bock H, Bodenmann D (2012) Inter-agency comparison of TanDEM-X baseline solutions. Advances in Space Research 50(2):260-271, DOI 10.1016/j.asr.2012.03.027

Jäggi A, Dahle C, Arnold D, Bock H, Meyer U, Beutler G, van den IJssel J (2016) Swarm kinematic orbits and gravity fields from 18 months of GPS data. Advances in Space Research 57(1):218-233, DOI 10.1016/j.asr.2015.10.035

Kang Z, Tapley B, Bettadpur S, Ries J, Nagel P, Pastor R (2006) Precise orbit determination for the GRACE mission using only GPS data. Journal of Geodesy 80(6):322-331, DOI 10.1007/ s00190-006-0073-5

Krieger G, Hajnsek I, Papathanassiou KP, Younis M, Moreira A (2010) Interferometric synthetic aperture radar (SAR) missions employing formation flying. Proceedings of the IEEE 98(5):816-843, DOI 10.1109/JPROC.2009.2038948

Kroes R, Montenbruck O, Bertiger W, Visser P (2005) Precise GRACE baseline determination using GPS. GPS Solutions 9(1):21-31, DOI 10.1007/s10291-004-0123-5

Lambin J, Morrow R, Fu LL, Willis JK, Bonekamp H, Lillibridge J, Perbos J, Zaouche G, Vaze P, Bannoura W, et al (2010) The OSTM/Jason-2 mission. Marine Geodesy 33(S1):4-25, DOI 10. 1080/01490419.2010.491030

Laurichesse D, Mercier F, Berthias JP, Broca P, Cerri L (2009) Integer ambiguity resolution on undifferenced GPS phase measurements and its application to PPP and satellite precise orbit determination. Navigation 56(2):135-149, DOI 10.1002/j.2161-4296.2009. tb01750.x

Loyer S, Perosanz F, Mercier F, Capdeville H, Marty JC (2012) Zero-difference GPS ambiguity resolution at CNES-CLS IGS analysis center. Journal of Geodesy 86(11):991, DOI 10.1007/ s00190-012-0559-2

Luthcke S, Zelensky N, Rowlands D, Lemoine F, Williams T (2003) The 1-centimeter orbit: Jason-1 precision orbit determination using GPS, SLR, DORIS, and altimeter data. Marine Geodesy 26(34):399-421, DOI 10.1080/714044529

Lyard F, Lefevre F, Letellier T, Francis O (2006) Modelling the global ocean tides: modern insights from FES2004. Ocean Dynamics 56(5-6):394-415, DOI 10.1007/s10236-006-0086-x

McWilliams HE (2015) Validating the 1-cm orbit, MSc thesis, university of texas at austin. URL https://repositories.lib. utexas.edu/handle/2152/34038

Mendes V, Pavlis E (2004) High-accuracy zenith delay prediction at optical wavelengths. Geophysical Research Letters 31(14):L14,602, DOI 10.1029/2004GL020308

Montenbruck O, Kroes R (2003) In-flight performance analysis of the CHAMP BlackJack GPS receiver. GPS solutions 7(2):74-86, DOI 10.1007/s10291-003-0055-5

Montenbruck O, Neubert R (2011) Range correction for the CryoSat and GOCE laser retroreflector arrays, DLR/GSOC TN 11-01. URL https://ilrs.cddis.eosdis.nasa.gov/docs/ TN_1101_IPIE_LRA_v1.0.pdf

Montenbruck O, Garcia-Fernandez M, Williams J (2006) Performance comparison of semicodeless GPS receivers for LEO satellites. GPS Solutions 10(4):249-261, DOI 10.1007/s10291-006-0025-9

Montenbruck O, Allende-Alba G, Rosello J, Tossaint M, Zangerl F (2017a) Precise orbit and baseline determination for the SAOCOM-CS bistatic radar mission. In: Proc. ION-ITM-2017, pp $123-131$

Montenbruck O, Hackel S, Jäggi A (2017b) Precise orbit determination of the Sentinel-3A altimetry satellite using ambiguity- fixed GPS carrier phase observations. Journal of Geodesy DOI $10.1007 / \mathrm{s} 00190-017-1090-2$

Neubert R (2009) The center of mass correction (CoM) for laser ranging data of the CHAMP reflector, issue c, 14 oct 2009. URL https://ilrs.cddis.eosdis.nasa.gov/docs/ CH_GRACE_COM_C.pdf

Neubert R, Grunwaldt L, Neubert J (1998) The retro-reflector for the CHAMP satellite: Final design and realization. In: Proceedings of the 11th International Workshop on Laser Ranging, pp 260-270

Otsubo T (2017) Multi-satellite bias analysis report v2 for worldwide satellite laser ranging stations. URL http://geo. science. hit-u.ac.jp/slr/bias/

Otsubo T, Kubo-oka T, Gotoh T, Ichikawa R (2004) Atmospheric "blue sky" effects on SLR station coordinates. In: Proc. 14th ILRS Workshop, San Feronando, Spain, pp 69-74

Pearlman MR, Degnan JJ, Bosworth J (2002) The International Laser Ranging Service. Advances in Space Research 30(2):135-143, DOI 10.1016/S0273-1177(02)00277-6

Peter H, Fernández J, Ayuga F, Féménias P (2016) Copernicus POD service: Orbit determination of the Sentinel satellites. In: EGU General Assembly Conference Abstracts, vol 18, p 3855

Peter H, Jäggi A, Fernández J, Escobar D, Ayuga F, Arnold D, Wermuth M, Hackel S, Otten M, Simons W, Visser P, Hugentobler U, Féménias P (2017) Sentinel-1A - first precise orbit determination results. Advances in Space Research DOI 10.1016/j.asr.2017.05. 034

Petit G, Luzum B (2010) IERS Conventions (2010). IERS Technical Note no. 36, Verlag des Bundesamts für Kartographie und Geodäsie, Frankfurt

Prange L, Jäggi A, Dach R, Bock H, Beutler G, Mervart L (2010) AIUB-CHAMP02S: The influence of GNSS model changes on gravity field recovery using spaceborne GPS. Advances in Space Research 45(2):215-224, DOI 10.1016/j.asr.2009.09.020

Quartly GD, Legeais JF, Ablain M, Zawadzki L, Joana Fernandes M, Rudenko S, Carrère L, Nilo García P, Cipollini P, Andersen OB, Poisson JC, Mbajon Njiche S, Cazenave A, Benveniste J (2017) A new phase in the production of quality-controlled sea level data. Earth System Science Data 9:557-572, DOI 10.5194/ essd-9-557-2017

Rebischung P (2012) IGb08: an update on IGS08, IGSMAIL6663, 24 Sep. 2012. URL https://igscb.jpl.nasa. gov/pipermail/igsmail/2012/007853.html

Rebischung P, Griffiths J, Ray J, Schmid R, Collilieux X, Garayt B (2012) IGS08: the IGS realization of ITRF2008. GPS Solutions 16(4):483-494, DOI 10.1007/s10291-011-0248-2

Reigber C, Lühr H, Schwintzer P (2000) Status of the CHAMP mission. In: Towards an Integrated Global Geodetic Observing System (IGGOS), Springer, pp 63-65

Ricklefs RL, Moore CJ (2008) Consolidated laser ranging data format (CRD), version 1.01. Format specification, ILRS Data Formats and Procedures Working Group, URL https://ilrs.cddis.eosdis.nasa.gov/docs/ 2009/crd_v1.01.pdf

Rim HJ, Yoon S, Schultz BE (2013) The GLAS algorithm theoretical basis document for precision orbit determination (POD). Technical Report NASA/TM-2013-208641, Volume 11, NASA Goddard Space Flight Center, URL https://nsidc.org/sites/nsidc.org/files/ technical-references/GLAS_ATBD_Precision_ Orbit_Determination_v11_03_2013.pdf

Roselló Guasch J, Silvestrin P, Aguirre M, Massotti L (2010) Navigation needs for ESA's earth observation missions. In: Small Satellite Missions for Earth Observation, Springer, pp 439-447

Schutz BE, Tapley BD, Abusali PAM, Rim HJ (1994) Dynamic orbit determination using GPS measurements from TOPEX/POSEIDON. Geophysical Research Letters 21(19):2179- 
2182

Schutz BE, Zwally HJ, Shuman CA, Hancock D, DiMarzio JP (2005) Overview of the ICESat mission. Geophysical Research Letters 32(21):1-4, DOI 10.1029/2005GL024009

Shargorodsky V (2002) CryoSat-LRR-01 laser retro reflector technical description, K01-E1095-00-00 TO, Scientific Research Institute for Precision Instruments, Moscow. URL https://ilrs.cddis.eosdis.nasa.gov/docs/ CRYOSAT_LRR_01_DATA_PACKAGE.pdf

Sośnica K, Thaller D, Dach R, Jäggi A, Beutler G (2013) Impact of loading displacements on SLR-derived parameters and on the consistency between GNSS and SLR results. Journal of Geodesy 87(8):751-769, DOI 10.1007/s00190-013-0644-1

Švehla D, Rothacher M (2002) Kinematic orbit determination of LEOs based on zero or double-difference algorithms using simulated and real SST GPS data. In: Vistas for geodesy in the new millennium, Springer, pp 322-328

Švehla D, Rothacher M (2003) CHAMP double-difference kinematic POD with ambiguity resolution. In: First CHAMP Mission Results for Gravity, Magnetic and Atmospheric Studies, Springer, pp 7077

Tapley B, Schutz B, Born GH (2004) Statistical orbit determination. Academic Press

Tapley BD, Bettadpur S, Ries JC, Thompson PF, Watkins MM (2004) GRACE measurements of mass variability in the Earth system. Science 305:503-506, DOI 10.1126/science.1099192

Wijaya DD, Böhm J, Karbon M, Kràsnà H, Schuh H (2013) Atmospheric pressure loading. In: Atmospheric Effects in Space Geodesy, Springer, pp 137-157, DOI 10.1007/978-3-642-36932-2_4

Wu SC, Yunck TP, Thornton CL (1991) Reduced-dynamic technique for precise orbit determination of low earth satellites. Journal of Guidance, Control, and Dynamics 14(1):24-30

Yoon S, Rim HJ, Schutz BE (2012) Phase center variation modeling of ICESat GPS antenna for precision orbit determination. In: AIAA/AAS Astrodynamics Specialist Conference, AIAA 20124879, pp 1-12

Yoon YT, Eineder M, Yague-Martinez N, Montenbruck O (2009) precise trajectory estimation and quality assessment. IEEE Transactions on Geoscience and Remote Sensing 47(6):1859-1868, DOI 10.1109/TGRS.2008.2006983

Yunck T, Bertiger W, Wu S, Bar-Sever Y, Christensen E, Haines B, Lichten S, Muellerschoen R, Vigue Y, Willis P (1994) First assessment of GPS-based reduced dynamic orbit determination on TOPEX/Poseidon. Geophysical Research Letters 21(7):541-544

Zangerl F, Griesauer F, Sust M, Montenbruck O, Buchert S, Garcia A (2014) SWARM GPS precise orbit determination receiver initial in-orbit performance evaluation. In: Proc. ION GNSS+2014

Zelensky NP, Lemoine FG, Beckley BD, Chinn DS, Pavlis DE (2018) Impact of ITRS 2014 realizations on altimeter satellite precise orbit determination. Advances in Space Research 61(1):45-73, DOI 10.1016/j.asr.2017.07.044

Zin A, Landenna S, Conti A (2006) Satellite to satellite tracking instrument. In: Proc. 3rd Int. GOCE User Workshop 